\title{
The Nature of the Steady State in Models of Optimal Growth Under Uncertainty*
}

\author{
Tapan Mitra†, Luigi Montrucchio ${ }^{\ddagger}$ and Fabio Privileggi ${ }^{\S}$
}

\begin{abstract}
We study a one-sector stochastic optimal growth model with a representative agent. Utility is logarithmic and the production function is of the Cobb-Douglas form with capital exponent $\alpha$. Production is affected by a multiplicative shock taking one of two values with positive probabilities $p$ and $1-p$. It is well known that for this economy, optimal paths converge to a unique steady state, which is an invariant distribution. We are concerned with properties of this distribution. By using the theory of Iterated Function Systems, we are able to characterize such a distribution in terms of singularity versus absolute continuity as parameters $\alpha$ and $p$ change. We establish mutual singularity of the invariant distributions as $p$ varies between 0 and 1 whenever $\alpha<1 / 2$. More delicate is the case $\alpha>1 / 2$. Singularity with respect to Lebesgue measure also appears for values $\alpha, p$ such that $\alpha<p^{p}(1-p)^{(1-p)}$. For $\alpha>p^{p}(1-p)^{(1-p)}$ and $1 / 3 \leq p \leq 2 / 3$, Peres and Solomyak (1998) have shown that the distribution is a.e. absolutely continuous. Characterization of the invariant distribution in the remaining cases is still an open question. The entire analysis is summarized through a bifurcation diagram, drawn in terms of pairs $(\alpha, p)$.
\end{abstract}

JEL Classification Numbers: C61, O41

*This research was partially supported by CNR (Italy) under the "Short-term mobility" program and by M.U.R.S.T. (Italy) National Group on "Nonlinear Dynamics and Stochastic Models in Economics and Finance". We are indebted to Rabi Bhattacharya for providing us with the reference to Solomyak's (1995) paper.

${ }^{\dagger}$ Dept. of Economics, Cornell University, Uris Hall, Ithaca, NY 14853 (USA); tm19@cornell.edu

${ }^{\ddagger}$ Dept. of Statistics and Mathematics, P. Arbarello 8, 10122 Torino (Italy); luigi.montrucchio@econ.unito.it

$\S$ Dept. of Public Policy and Public Choice, C. Borsalino 50, 15100 Alessandria (Italy); privileg@unipmn.it 


\section{Introduction}

In deterministic optimal growth models, it is customary to characterize a (stable) steady state, and also to examine how the steady state changes as parameters of the model change.

In contrast to this, relatively little is known about the nature of the steady state of stochastic optimal growth models. A stochastic steady state is identified as an invariant distribution, and, since the seminal papers by Lucas-Prescott [39] and Brock-Mirman [10], a large part of the literature on the subject has focused on the existence, uniqueness and global stability of this invariant distribution. See, for example, [43], [44], [9], [14], [26] and, more recently, [32] and [40].

However, the problem of characterizing such a distribution appears to have received far less attention even for the simplest examples arising in such models. Consequently, our state of knowledge, regarding how the stochastic steady state changes with the parameters of the model, is extremely limited. An exception is the paper by Mirman and Zilcha [45] where, for some examples with specific parameter values, the limiting distribution function can be directly computed.

The aim of the present work is to examine one example of an optimal growth model under uncertainty, in as much detail as possible, and address specifically two questions regarding the stochastic steady state of the model:

1. What is the nature of the invariant distribution representing the stochastic steady-state? Specifically, is it absolutely continuous, so that it can be represented by a density, which could possibly be estimated in terms of a few parameters? Or, is it singular, so that, essentially, we do not have a convenient way of representing the function without actually stating the value of the function for every point in its domain?

2. How does the nature of the invariant distribution (specifically, its absolute continuity or singularity) change with parameters of the optimal growth model generating it? Specifically, is it possible to represent this information in the form of a convenient "bifurcation diagram" involving the key parameters of the optimal growth model?

These questions appear to be quite basic. Yet, as we will see, answering them, even in the context of a simple example of an optimal growth model, is surprisingly difficult, and the whole 
family of invariant measures obtained for a large set of parameters values exhibits an extremely rich and complicated structure.

The particular example of optimal growth under uncertainty that we discuss is a familiar one in which the utility function is logarithmic and the production function is of the Cobb-Douglas form with capital exponent $\alpha$. Uncertainty is captured in terms of a multiplicative shock to the production function taking one of two values, with positive probabilities $p$ and $1-p$, which are fixed over time. [That is, the process of exogenous shocks is independent and identically distributed with marginal probability distribution given by these specified probabilities].

It is known that this optimal growth problem can be solved, using dynamic programming methods, to obtain an optimal policy function. This, in turn, yields a non-linear stochastic process, governing the evolution of the state variable (the capital stock of the economy). Using the specific structure of the example, it is possible, through an appropriate log-transformation, to convert this into an iterated function system (IFS) involving affine functions (or similarities). A straightforward application of the theory of iterated function systems leads to the functional equation governing the invariant distribution ${ }^{1}$ of the (transformed) random dynamical system.

We note that since our random dynamical system is generated by a finite number of affine maps which are contractions, the existence, uniqueness and global stability of the invariant distribution (starting from an arbitrary initial distribution), which the literature is principally concerned with, are guaranteed by a straightforward application of the standard theory of iterated function systems, developed by Karlin [36], Norman [47], Hutchinson [33], Barnsley and Demko [4], and others. [For ease of exposition, a brief review of the theory of IFS is presented in Section 2].

Our objective is to be able to say something useful about the stochastic steady state beyond the above mentioned properties ${ }^{2}$. To this end, we provide a heuristic discussion in Section 3 regarding the features of the stochastic steady state that one may expect to obtain. In particular, we observe that the support of the invariant measure should be "full" or "thin" depending on whether or not the images of the maps of our dynamical system "overlap". In addition, we relate the invariant measure of our IFS to the distribution of a random variable,

\footnotetext{
${ }^{1}$ Whenever the IFS involves similarities, its invariant distribution is also called a self-similar measure.

${ }^{2}$ The idea of characterizing complexity of measures, usually, but not necessarily, defined over fractal supports, is due to Mandelbrot [41], who called this approach multifractal analysis. Far from pursuing a completely exhaustive multifractal study, here we confine our research on singularity versus absolute continuity properties of the invariant distribution.
} 
defined in terms of an infinite series, and known as an Erdös series. This link allows us to exploit some of the mathematical literature on the distribution of the Erdös series, which we review briefly.

In Section 4, we start our formal analysis of the nature of the stochastic steady state. In the first subsection we confine our attention to "low" values of our technological parameter, $\alpha$, which in our case turns out to be the range $\alpha \in(0,1 / 2)$. For $\alpha \in(0,1 / 2)$, the images of the maps of our IFS do not overlap, so that the support of the invariant distribution is seen to be a Cantor-like set of Lebesgue measure zero. The invariant distribution is clearly singular in this case, and its graph is the well-known "Devil's Staircase". It is worthwhile to note that the actual magnitude of the probability of picking each map of the IFS plays no role in obtaining this conclusion.

In contrast, for the borderline case of $\alpha=1 / 2$, the magnitude of the probability of picking each map of the IFS plays a crucial role in determining the nature of the stochastic steady state. Thus, in the equal probability case, it is easy to see that the invariant distribution is uniform and so absolutely continuous. On the other hand, for the unequal probability case, the invariant distribution is always singular, leading to a continuous, strictly increasing distribution function on the unit interval, whose derivative is zero (Lebesgue) almost everywhere. Construction of a function with these features figures prominently in Lebesgue's theory of integration.

To conclude Section 4, we establish an even stronger property of the invariant distributions for $\alpha \in(0,1 / 2]$. We prove that they are all mutually singular, that is, they concentrate over subsets of Cantor-like sets that have empty intersection. This result is in line with the work started by Montrucchio and Privileggi [46], where they found that the support of the invariant distribution is a fractal set (for $\alpha \in(0,1 / 2)$ ). Thus rather strange dynamics can be optimal in standard neo-classical stochastic growth models. The "mutual singularity" property shows that the "thin support" of the invariant distribution is itself highly sensitive to small changes in probabilities.

Section 5 provides a further refinement of the singularity result by showing that for the combination of parameter values $(\alpha, p)$ for which

$$
1 / 2<\alpha<p^{p}(1-p)^{(1-p)}
$$

the invariant distribution is singular with respect to Lebesgue measure. This means that the limiting distribution can be singular for (a set of positive Lebesgue measure of) values $\alpha>1 / 2$ 
as well, provided that probabilities $p$ and $1-p$ are asymmetric enough. Coupled with singularity for $\alpha \in(0,1 / 2)$, all $p$, and for $\alpha=1 / 2, p \neq 1 / 2$, this is a well known result in the mathematical literature (see the discussion in Section 5). Here, we provide a simplified proof based on the concept of Hausdorff dimension, which we discuss briefly to keep our exposition self-contained.

In Section 6, we turn our attention to the remaining cases in which the technological parameter, $\alpha$, is relatively "high" and probabilities $p$ and $1-p$ are not too asymmetric, that is, pairs of parameters $(\alpha, p)$ such that

$$
p^{p}(1-p)^{(1-p)}<\alpha<1
$$

This turns out to be the most difficult situation to analyze. Our investigation of this case is incomplete; we report an important result, recently established by Peres and Solomyak [51], which establishes almost everywhere absolute continuity of the invariant distribution for values of the probability $p$ in the interval $[1 / 3,2 / 3]$ and pairs $(\alpha, p)$ satisfying (1).

It is interesting to find that the function $p^{p}(1-p)^{(1-p)}$, at least for $1 / 3 \leq p \leq 2 / 3$, turns out to be the borderline between singularity and (generic) absolute continuity of the invariant distribution. In particular, for a given $p$ in $[1 / 3,2 / 3]$, as $\alpha$ increases from $1 / 2$ to 1 (which means that the degree of "overlapping" of the images of the maps of the IFS increases), the distribution turns from a singular one to an absolutely continuous one around the value $\alpha=p^{p}(1-p)^{(1-p)}$. That is, the turning point from singularity to absolute continuity of the invariant distribution is determined precisely by the entropy, $[-p \ln p-(1-p) \ln (1-p)]$, of the Bernoulli process underlying the exogenous shocks in our growth model. Furthermore, invariant distributions exhibit increasing "smoothness" above the entropy curve: let $1<\gamma \leq 2$, then for almost every $p^{p}(1-p)^{(1-p)}<\alpha \leq p^{2}+(1-p)^{2}$ the distributions have densities in $L^{\gamma}$, with $\gamma$ increasing as $\alpha$ increases.

The whole discussion leads to Section 7, where a partial "bifurcation diagram" in terms of the parameters $(\alpha, p)$ on the unit square, indicates how the nature of the stochastic steady state varies with changes in these parameters (see Figure 5). By analyzing another result by Peres and Solomyak [51], which establishes a necessary smoothness condition for the invariant probability, we discuss further developments and we sketch a conjectured "complete" bifurcation diagram (see Figure 6). 


\section{The Framework}

In this section, we will describe the framework for our subsequent analysis. The concepts and basic results of iterated function systems (IFS) are essential to this study, and we provide a simplified exposition in Section 2.1. This exposition is based on Stark and Bressloff [56], and Lasota and Mackey [37]. In Section 2.2, we introduce the familiar model of optimal growth under uncertainty, and show, in the context of a particular example, how the analysis of optimal dynamics in this model reduces to the study of an IFS.

\subsection{Iterated Function Systems}

\subsubsection{Definitions}

Let $(X, d)$ be a complete metric space. An IFS $(f, p)$ consists of a finite number of mappings $\left\{f_{0}, \ldots, f_{m-1}\right\}$ from $X$ to $X$, and a set of associated probabilities $\left\{p_{0}, \ldots, p_{m-1}\right\}$ (so that $p_{z}>0$ for $z=0, \ldots, m-1$ and $p_{0}+\cdots+p_{m-1}=1$ ). Such an IFS gives a (random) dynamical system by defining a (random) orbit of $f$ to be a sequence $\left\{x_{t}\right\}$ such that $x_{t+1}=f_{z_{t}}\left(x_{t}\right)$ where $z_{t}$ is chosen randomly from $\{0, \ldots, m-1\}$ for each $t$ with probability $p_{z_{t}}$.

We are interested in IFS with contracting maps. Hence, the $f_{z} \mathrm{~s}$ will be assumed to be contractions; that is, for each $z \in\{0, \ldots, m-1\}$ there is a $\gamma_{z}$ such that $0 \leq \gamma_{z}<1$ and for all $x, y \in X$

$$
d\left(f_{z}(x), f_{z}(y)\right) \leq \gamma_{z} d(x, y)
$$

We shall denote by $\gamma$ the maximum of the $\gamma_{z}$. Clearly $\gamma<1$.

The maps $\left\{f_{0}, \ldots, f_{m-1}\right\}$ induce an operator $T$ on $X$, called the Barnsley operator, defined by:

$$
T(A)=f_{0}(A) \cup \cdots \cup f_{m-1}(A), \quad A \subset X,
$$

where $f_{z}(A)$ denotes the image of the set $A$ through $f_{z}$. Operator $T$ allows for a definition of an invariant set of an IFS. A set $A_{f} \subset X$ is said to be an invariant set of $f$ if it is compact and satisfies

$$
T\left(A_{f}\right)=A_{f}
$$

One can also define a Markov operator $M$ which describes the evolution of probabilities under the process $x_{t+1}=f_{z_{t}}\left(x_{t}\right)$. Thus let $\mathcal{B}(X)$ be the $\sigma$-algebra of Borel measurable subsets of $X$ and $\mathcal{P}(X)$ the space of probability measures on $\mathcal{B}(X)$. Then if $\mu \in \mathcal{P}(X)$ is an initial 
probability distribution we want $M(\mu)$ to be the distribution after one iteration of $f$. $M$ is then given by

$$
M \mu(B)=\sum_{z=0}^{m-1} p_{z} \mu\left(f_{z}^{-1}(B)\right), \quad \text { for all } B \in \mathcal{B}(X)
$$

where $f_{z}^{-1}(B)$ denotes the set $\left\{x \in X: f_{z}(x) \in B\right\}$. Note that $M$ is a linear operator from $\mathcal{P}(X)$ to itself.

\subsubsection{The Invariant Measure of an IFS}

We shall be interested in the asymptotic behavior of measures under repeated application of $M$; in other words, in the long run behavior of distributions as they evolve under $f$. Since the maps $f_{z}$ of the IFS we are focusing on are contractions, it turns out that there is a unique invariant distribution $\mu_{f}$, that is

$$
\mu_{f}=M\left(\mu_{f}\right)
$$

and the iterates $M^{t}(\mu)$ s converge weakly to $\mu_{f}$ as $t \rightarrow \infty$ for all initial distributions $\mu \in \mathcal{P}(X)$.

The simplest proof ${ }^{3}$ of the existence and uniqueness of $\mu_{f}$ uses the Hutchinson [33] metric $L$ defined over a subset $\mathcal{L}(X)$ of $\mathcal{P}(X)$. For all pairs $\mu, v \in \mathcal{P}(X)$, define a pseudo-metric ${ }^{4}$ by

$$
L(\mu, v)=\sup \left\{\left|\int_{X} \phi d \mu-\int_{X} \phi d v\right|: \operatorname{Lip}(\phi) \leq 1\right\}
$$

where the supremum is taken over all functions $\phi: X \rightarrow \mathbb{R}$ that are bounded and with Lipschitz constant not larger than 1 . Fix any $a \in X$, and define ${ }^{5}$

$$
\mathcal{L}(X)=\left\{\mu \in \mathcal{P}(X): \int d(x, a) d \mu<\infty\right\} .
$$

$L$ defined in (6) satisfies the triangle inequality, thus $\mathcal{L}(X)$ does not depend on $a$.

When $f$ satisfies $(2)$, the operator $M: \mathcal{L}(X) \rightarrow \mathcal{L}(X)$ turns out to be a contraction with respect to $L$. In fact we have $L(M(\mu), M(v)) \leq \gamma L(\mu, v)$. Then through a contraction mapping

\footnotetext{
${ }^{3}$ Hutchinson's [33] construction works for the general case of an IFS with contracting maps. However, refinements of the theory are possible for special classes of IFS. Karlin [36] proves existence of the invariant measure for the special category of affine maps, while allowing the probabilities to depend on the state and be Markovian.

${ }^{4}$ This is not a metric: $L$ is clearly non-negative, symmetric and satisfies the triangle inequality, but it could be plus infinity for some pair $\mu, v \in \mathcal{P}(X)$; also $L(\mu, v)=0$ does not necessarily imply $\mu=v$. Therefore, we need to restrict our probability space to a subset $\mathcal{L}(X)$.

${ }^{5}$ Note that the space $\mathcal{L}(X)$ is more general than the space of probability measures with bounded support actually used by Hutchinson [33].
} 
argument it can be shown that $\mu_{f}$ is the unique attracting fixed point of $M$. Since the $L$-metric convergence implies weak convergence over $\mathcal{L}(X)$ (see, Proposition 5 in the Appendix), it follows that $M^{t}(\mu)$ converges weakly to $\mu_{f}$.

Since the measure $\mu_{f}$ is the unique invariant measure for $M$, it must be ergodic. However a much stronger property holds: $\mu_{f}$ is in fact a Sinai-Ruelle-Bowen (SRB) measure. This means that for almost every sequence $\left\{f_{z_{t}}\right\}$ of maps the visiting frequency of the orbit $\left\{x_{t}\right\}$ to a set $B \in \mathcal{B}(X)$ is simply $\mu_{f}(B)$, that is

$$
\lim _{n \rightarrow \infty} \frac{\#\left\{x_{t} \in B: 1 \leq t \leq n\right\}}{n}=\mu_{f}(B)
$$

This was first proved by Norman [47] and later independently by Elton [19].

Equation (7) provides the simplest method of drawing $\mu_{f}$.

\subsubsection{The Attractor of an IFS}

The existence of a (unique) invariant set, $A_{f}$, of $f$ can be seen as follows. Consider the space $\mathcal{K}(X)$, the set of all non-empty compact sets in $X$. This is itself a complete metric space when endowed with the Hausdorff metric $d_{H}$, which is defined (see Falconer [23]) as follows. Let

$$
A_{\delta}=\{x \in X: d(x-a)<\delta \text { for some } a \in A\}
$$

denote the $\delta$-parallel body of $A \in \mathcal{K}(X)$, i.e., the set of points within distance $\delta$ of $A$. Then the Hausdorff metric is defined for $A, B \in \mathcal{K}(X)$ as

$$
d_{H}(A, B)=\inf \left\{\delta>0: A \subset B_{\delta} \text { and } B \subset A_{\delta}\right\}
$$

It is easily seen that $d_{H}(T(A), T(B)) \leq \gamma d_{H}(A, B)$ (see, for example [23] or [37]), so that $T$ is a contraction on $\mathcal{K}(X)$. Thus, $T$ has a unique fixed point, call it $A_{f}$. Then $A_{f}$ is the unique invariant set of $f$. Further, we have $T^{t}\left(A_{0}\right) \rightarrow A_{f}$ as $t \rightarrow \infty$ for any initial $A_{0} \in \mathcal{K}(X)$; and, given any $A_{0} \in \mathcal{K}(X)$ such that $T\left(A_{0}\right) \subset A_{0}$, we have $A_{f} \subset T^{t+1}\left(A_{0}\right) \subset T^{t}\left(A_{0}\right)$ for $t=0,1,2, \ldots$ (see Proposition 12.8.3, p. 439, of Lasota and Mackey [37]).

The support of the invariant distribution, $\mu_{f}$, is called the attractor of $f$. It is useful to note that this attractor is the same as the unique invariant set, $A_{f}$, obtained (above) by purely non-probabilistic methods. This can be seen as follows: given any $\mu \in \mathcal{P}(X)$, define $\mu_{t}=M^{t}(\mu)$ for $t=0,1,2, \ldots$. Then, if the support of $\mu$ is a compact set, we have (by Proposition 12.8.2, p. 435 of Lasota and Mackey [37]) (support of $\mu_{t}$ ) $=T^{t}$ (support of $\mu$ ). Applying this result to 
$\mu_{f}$, we have (support of $\left.\mu_{f}\right)=T$ (support of $\mu_{f}$ ), so that (since $A_{f}$ is the unique fixed point of $T)$, (support of $\left.\mu_{f}\right)=A_{f}$.

\subsection{One Sector Log-Cobb-Douglas Optimal Growth}

Let us recall the formulation of the one-sector growth model with a Cobb-Douglas production function $G(x)=x^{\alpha}, 0<\alpha<1$, with a representative decision maker's utility given by $u(c)=$ $\ln c$. Suppose that an exogenous perturbation may reduce ${ }^{6}$ production by some parameter $0<k<1$ with probability $p>0$ (the same for all $t=0,1, \ldots$ ). This independent and identically distributed random shock enters multiplicatively into the production process so that output is given by $G_{r}(x)=r x^{\alpha}$ where $r \in\{k, 1\}$. The dynamic optimization problem can be explicitly written as follows:

$$
\max \mathbb{E}_{0} \sum_{t=0}^{\infty} \beta^{t} \ln c_{t}
$$

where $0<\beta<1$ is the discount factor, and the maximization is over all consumption plans $c=c_{0}, c_{1}, \ldots$ such that for $t=0,1,2, \ldots$

$$
c_{t}=r_{t} x_{t}^{\alpha}-x_{t+1}, \quad c_{t} \geq 0, \quad x_{t} \geq 0
$$

and $x_{0}, r_{0}$ are given.

It is well known that the optimal policy for the concave problem just described is $g(x, z)=$ $\alpha \beta r x^{\alpha}$ (see [57]); i.e. the plan $x_{t}$ generated recursively by

$$
x_{t+1}=g\left(x_{t}, r_{t}\right)=\alpha \beta r_{t} x_{t}^{\alpha}
$$

is optimal.

Consider now the random dynamical system obtained by the following logarithmic transformation of $x_{t}$ :

$$
y_{t}=-\frac{1-\alpha}{\ln k} \ln x_{t}+1+\frac{\ln \alpha+\ln \beta}{\ln k} .
$$

The new variable $y_{t}$, associated with $x_{t}$, evolves according to a linear policy, so that

$$
y_{t+1}=\alpha y_{t}+(1-\alpha)\left(1-\frac{\ln r_{t}}{\ln k}\right)
$$

which can be rewritten as

$$
\begin{cases}y_{t+1}=\alpha y_{t} & \text { with probability } p \\ y_{t+1}=\alpha y_{t}+(1-\alpha) & \text { with probability } 1-p\end{cases}
$$

\footnotetext{
${ }^{6}$ The same framework works for a perturbation that "increases" production: $k>1$.
} 
Define the maps $f_{0}, f_{1}$ from $[0,1]$ to $[0,1]$ by:

$$
\left\{\begin{array}{l}
f_{0}(y)=\alpha y \\
f_{1}(y)=\alpha y+(1-\alpha) .
\end{array}\right.
$$

It is useful to note here that the map $f_{0}$ corresponds to the case where the shock, $r$, takes the value $k$; and the map $f_{1}$ corresponds to the case where the shock, $r$, takes the value 1 . Denote $(p, 1-p)$ by $\left(p_{0}, p_{1}\right)$. Then $\left(f_{0}, f_{1}\right)$, together with $\left(p_{0}, p_{1}\right)$ is an iterated function system over the interval $[0,1]$. The maps $f_{z}$, for $z \in\{0,1\}$, are clearly affine (or similarities, as they are one-dimensional) and since $0<\alpha<1$, they are contractions on [0,1]. Thus, we can apply the methods of Section 2.1 directly to our IFS, and obtain the following proposition, which summarizes for future reference the main results regarding the invariant distribution associated with our IFS.

Proposition 1 Consider the IFS described by $[0,1],\left(f_{0}, f_{1}\right),\left(p_{0}, p_{1}\right)$.

i) There is a unique compact set $A_{f} \subset[0,1]$, such that $f_{0}\left(A_{f}\right) \cup f_{1}\left(A_{f}\right)=A_{f}$.

ii) For any compact set $A$, denoting $A_{t}=T^{t}(A)$ for $t=0,1,2, \ldots$, we have $A_{0} \supset A_{1} \supset A_{2} \supset$ $\ldots \supset A_{f}$ whenever $T(A) \subset A$.

iii) There is a unique probability distribution $\mu_{f}$ on $([0,1], \mathcal{B}([0,1]))$ satisfying the functional equation (5), that is,

$$
\mu_{f}(A)=p_{0} \mu_{f}\left(f_{0}^{-1}(A)\right)+p_{1} \mu_{f}\left(f_{1}^{-1}(A)\right) \quad \text { for all } A \in \mathcal{B}([0,1])
$$

Further, $A_{f}$ is the support of $\mu_{f}$.

iv) For $\mu \in \mathcal{L}([0,1])$, denoting $\mu_{t}=M^{t}(\mu)$ for $t=0,1,2, \ldots, L\left(\mu_{t}, \mu_{f}\right) \rightarrow 0$ as $t \rightarrow \infty$, and further $\mu_{t}$ converges weakly to $\mu_{f}$.

\section{Nature of the Stochastic Steady State: Discussion}

In this section, we provide a preliminary discussion on the nature of the stochastic steady state (invariant distribution) obtained in Section 2. This heuristic material helps us to proceed with the formal presentation of the main results of the paper in the next three sections. 
Our discussion pertains to three sub-topics. First, we comment on the support of the invariant distribution, and how this is related to "overlaps", or lack of it, of the IFS. Second, we relate the study of our iterated function system to the analysis of a certain infinite series, which we refer to as the Erdös series. Third, we summarize what is known in the mathematics literature regarding the behavior of the Erdös series (equivalently the iterated function system).

\subsection{The Support of the Invariant Distribution}

The graphs of the functions given by (9), show that for $0<\alpha<1 / 2$, the image sets of the two functions $f_{0}$ and $f_{1}$ are disjoint, a situation which can be described as the "non-overlapping" case (see Figure 1(a)). In this case, the "gap" between the two image sets (in the unit interval) will "spread" through the unit interval by successive applications of the maps (9). Thus, one would expect the support of the invariant distribution to be "thin" (with zero Lebesgue measure) and have features of the usual Cantor ternary set; in fact, for $\alpha=1 / 3$, the support is precisely the Cantor ternary set.

On the other hand, for $1 / 2 \leq \alpha<1$, the image sets of the functions $f_{0}$ and $f_{1}$ have a non-empty intersection. We can refer to this as the "overlapping" case (see Figure 1(b)). Here, the successive iterations of the overlap can be expected to "fill up" the unit interval, so the invariant distribution should have full support.

The above heuristics are actually seen to be valid as we demonstrate in the following sections.

It is important to remark that this construction does not depend on the magnitude of the discount factor $\beta$ nor on the amplitude of the shock $k$, but only on the technological parameter $\alpha$. The discount factor $\beta$ only shifts the support of the invariant distribution of the original model over the real line, while the exogenous shock $k$ affects its amplitude.

\subsection{Erdös Series}

Let $\Omega$ be the space of all infinite sequences of zeros and ones; that is, an element of $\Omega$ is $\omega=\left(\omega_{0}, \omega_{1}, \omega_{2}, \ldots\right)$ where $\omega_{t} \in\{0,1\}$ for $t=0,1, \ldots$ Let $\mathcal{F}$ be the smallest $\sigma$-algebra containing all the finite $n$-cylinders $\left(\omega_{0}, \omega_{1}, \ldots, \omega_{n-1}\right) \times\{0,1\} \times\{0,1\} \times \cdots$ and $P$ the product measure over $\mathcal{F}$ generated by the probability over $\{0,1\}$ such that $\operatorname{Pr}(0)=p$ and $\operatorname{Pr}(1)=1-p$. Then we have a probability space $(\Omega, \mathcal{F}, P)$ which we refer to as a Bernoulli space. Define the projection $\varepsilon_{t}(\omega)=\omega_{t}$. Then $\left\{\omega_{t}\right\}$ is a sequence of independent and identically distributed 
random variables on $(\Omega, \mathcal{F}, P)$.

Using the sequence $\left\{\omega_{t}\right\}$, we can write our iterated function system as:

$$
y_{t+1}(\omega)=\alpha y_{t}(\omega)+(1-\alpha) \omega_{t}
$$

By Proposition 1, there is a unique limiting distribution $\mu_{f}$ to which the random variable $y_{t}(\omega)$ converges weakly, starting from any initial probability over $y_{0}(\omega)$. For simplicity, consider the Dirac measure that concentrates the whole probability over $y_{0}(\omega)=0$. Iteration of (10) leads to

$$
y_{t}(\omega)=(1-\alpha) \sum_{s=0}^{t-1} \omega_{s} \alpha^{t-s-1} .
$$

Since $\omega_{s}$ are independent random variables, the probability distribution of $y_{t}(\omega)$ is the same as that of the random variable

$$
(1-\alpha) \sum_{s=0}^{t-1} \omega_{s} \alpha^{s},
$$

which converges almost everywhere to the series

$$
\Pi_{\alpha}(\omega)=(1-\alpha) \sum_{s=0}^{\infty} \omega_{s} \alpha^{s}
$$

which is called Erdös series. Since almost everywhere convergence implies weak convergence (see, e.g., Theorem 25.2, p. 284 in [8]), the distribution of (11) is the same as the invariant distribution, $\mu_{f}$, of the system (10).

Note that this construction allows us to interpret $\mu_{f}$ as the image probability of $P$ through the $\operatorname{map} \Pi_{\alpha}: \Omega \rightarrow[0,1]$.

The Erdös series (11) is usually written in a slightly different format:

$$
\sum_{s=0}^{\infty} \pm \alpha^{s}
$$

it being understood that the minus sign is taken with probability $p$ and the plus sign with probability $(1-p)$. Note that $(12)$ is the same as $(11)$ where random variables $\omega_{t}^{\prime} \in\{-1,1\}$ are used in place of $\omega_{t} \in\{0,1\}$ and the image set of the corresponding IFS is the translation of interval $[0,1]$ to the interval $[-1 /(1-\alpha), 1 /(1-\alpha)]$. Thus, the nature of the distribution $\mu_{f}$ of (11) can be studied by analyzing the distribution of the random variable (12). 


\subsection{Brief Review of the Mathematical Literature}

Until a few years ago, the mathematical literature focused on the problem of analyzing the nature of the distribution of the random variable given in (12) exclusively in the special case where $p=1 / 2$. The topic is known as the study of "symmetric infinite Bernoulli convolutions". Only very recently some important results have been extended to the asymmetric case $p \neq 1 / 2$. All this stream of research has been striving around the fundamental question on deciding for what values of $\alpha, \mu_{f}$ is absolutely continuous, and for what values of $\alpha, \mu_{f}$ is singular. For an exhaustive mathematical survey on the whole history of Bernoulli convolutions, see [49].

It is known, in the symmetric case, that the distribution function is "pure"; that is, it is either absolutely continuous or it is singular (Jessen and Wintner [34]). Further, Kershner and Wintner [35] have shown that if $0<\alpha<1 / 2$, the support of the distribution function is a Cantor set of Lebesgue measure zero, and therefore the distribution function is singular. For $\alpha=1 / 2$, one gets the uniform distribution, which is not singular.

For the symmetric case $p=1 / 2$, denote by $S_{\perp}$ the set of $\alpha \in(1 / 2,1)$ such that $\mu_{f}$ is singular. It was often conjectured that the distribution function should be absolutely continuous with respect to Lebesgue measure when $1 / 2<\alpha<1$. Wintner [60] showed that if $\alpha$ is of the form $(1 / 2)^{1 / k}$ where $k \in\{1,2,3, \ldots\}$, then the distribution function is absolutely continuous. However, in the other direction, Erdös [20] showed that when $\alpha$ is the positive solution of the equation $\alpha^{2}+\alpha-1=0$, so that $\alpha=(\sqrt{5}-1) / 2$, then $\alpha \in S_{\perp}$. More generally, the results of Erdös [20] may be described as follows. Recall that an algebraic number is the solution of a polynomial, all of whose coefficients are integers, and whose leading coefficient is either 1 or -1 . An algebraic number is called a Pisot-Vijayaraghavan number when all its Galois conjugates are less than one in absolute value. Erdös [20] showed that when $\alpha$ is the reciprocal of a Pisot-Vijayaraghavan number, then $\alpha \in S_{\perp}$. It is an open question whether these numbers, which form a closed countable set, constitute all of $S_{\perp}$.

Erdös [21] also showed that $S_{\perp} \cap(\xi, 1)$ has zero Lebesgue measure for some $\xi<1$, so that absolute continuity of the invariant distribution obtains for (almost every) $\alpha$ sufficiently close to 1. A conjecture that emerged from these findings is that the set $S_{\perp}$ itself should have Lebesgue measure zero.

Since the contributions of Erdös, there were a few additional results for the case $1 / 2<$ $\alpha<1$, which are surveyed in Garsia [27]. In their brief discussion of this problem, Dubins and Freedman [15] state that deciding whether the distribution is singular or absolutely continuous 
for $\alpha>1 / 2$ is a "famous open question". And Edgar [17], briefly surveying this topic, refers to it as an "Erdös problem".

Solomyak [55] made a real breakthrough when he showed that $S_{\perp}$ has zero Lebesgue measure. More precisely, he established that for almost every $\alpha \in(1 / 2,1)$, the distribution has density in $L^{2}(\mathbb{R})$ and for almost every $\alpha \in\left(2^{-1 / 2}, 1\right)$ the density is bounded and continuous. A simpler proof of the same result was subsequently found by Peres and Solomyak [50]. A stronger result has been very lately achieved by Peres and Schlag [48], where they prove that the Hausdorff dimension (see Section 5.1 in the sequel) of the set $S_{\perp} \cap(\alpha, 1)$ is less than one for all $1 / 2<\alpha<1$. Note, however, that this still does not settle Garsia's [27] conjecture that the distribution is absolutely continuous for all but a countable number of values of $\alpha$ in $(1 / 2,1)$.

Very recent contributions to this literature deal with the asymmetric case $p \neq 1 / 2$. Mauldin and Simon [42] extended Jessen and Wintner's [34] finding on "purity" of the distribution function also for $p \neq 1 / 2$. Peres, Schlag and Solomyak [49] further pushed the argument to finite families of contracting similitudes (namely, IFS with $m \geq 2$ affine maps) by showing that if the limiting distribution $\nu$ is not singular, then it is absolutely continuous with respect to Lebesgue measure and the restriction of Lebesgue measure to the support of $\nu$ is absolutely continuous with respect to $\nu$.

Especially useful for our purposes, is the contribution by Peres and Solomyak [51], established for a generic family of contracting similitudes. We restate here their result in terms of our IFS given by (9). First, they found that $\mu_{f}$ is singular below the "entropy" curve, that is for values of parameters $(\alpha, p)$ such that $0<\alpha<p^{p}(1-p)^{(1-p)}$, while it is absolutely continuous for almost every $p^{p}(1-p)^{(1-p)}<\alpha<1$ whenever $1 / 3 \leq p \leq 2 / 3$. Moreover, for $1 / 3 \leq p \leq 2 / 3$, letting $1<\gamma \leq 2$, for almost every $\left[p^{\gamma}+(1-p)^{\gamma}\right]^{1 /(\gamma-1)} \leq \alpha<1, \mu_{f}$ has density in $L^{\gamma}$. Finally, this property is further refined by the following necessary smoothness condition: for all $p$ and all $\gamma>1$, if $\mu_{f}$ is absolutely continuous with density in $L^{\gamma}$, then $\alpha \geq\left[p^{\gamma}+(1-p)^{\gamma}\right]^{1 /(\gamma-1)}$. We shall discuss in more detail these last findings in Section 6.

\section{Singular Invariant Distributions}

In this section, we provide an analysis of the invariant distributions when the technological parameter, $\alpha$, is "low", which in our case translates to $\alpha \in(0,1 / 2]$. Thus, our results may be viewed as extensions of the mathematical literature reviewed in Section 3.3 to the case where 
$p$ is not necessarily equal to $1 / 2$.

In Section 4.1, we briefly review the case where $\alpha \in(0,1 / 2)$. Here, the theory for $p \neq 1 / 2$ is entirely analogous to the theory for $p=1 / 2$, since, as we have noted before, the support of the invariant distribution is a Cantor set of Lebesgue measure zero, and this support is independent of the magnitude of $p$.

In Section 4.2, we consider the "borderline" case where $\alpha=1 / 2$. Here the theory for $p \neq 1 / 2$ is entirely unlike the theory for $p=1 / 2$. While $p=1 / 2$ generates the uniform invariant distribution (which is, of course, absolutely continuous), $p \neq 1 / 2$ always generates a singular invariant distribution. However, unlike in Section 4.1, this singular distribution has full support.

Section 4.3 is devoted to an improvement of the existing theory. By using the relationship with Erdös series discussed in Section 3.2, we show that, for the whole range $\alpha \in(0,1 / 2]$, not only are the invariant measures singular with respect to Lebesgue measure, but they are also mutually singular. This, of course, indicates a high sensitivity of the limiting distribution with respect to changes in the parameter $p$. When $\alpha \in(0,1 / 2)$, not only does the support (attractor) exhibit fractal features, but also measures with the same support concentrate over sets with empty intersection for different probabilities $p$ and $p^{\prime}$.

\subsection{Singular Distributions of the Cantor Type}

Consider the case where the parameter, $\alpha$, has a value in $(0,1 / 2)$. Recall the Barnsley operator (3) and define

$$
A_{0}=[0,1], \quad \text { and } A_{n+1}=T\left(A_{n}\right) \quad \text { for } n=0,1, \ldots
$$

Then, we have

$$
\begin{aligned}
A_{1}=T\left(A_{0}\right)= & {[0, \alpha] \cup[1-\alpha, 1], } \\
A_{2}=T\left(A_{1}\right)= & {\left[0, \alpha^{2}\right] \cup\left[(1-\alpha),(1-\alpha)+\alpha^{2}\right] } \\
& \cup[\alpha(1-\alpha), \alpha] \cup[(1-\alpha)+\alpha(1-\alpha),(1-\alpha)+\alpha] .
\end{aligned}
$$

Note that the Lebesgue measure of $A_{0}$ is 1 , that of $A_{1}$ is $2 \alpha$, and that of $A_{2}$ is $2^{2} \alpha^{2}$. By induction, one can verify that $A_{n}=T^{n}([0,1])$ consists of $2^{n}$ intervals, each of Lebesgue measure $\alpha^{n}$, so that $\lambda\left(A_{n}\right)=2^{n} \alpha^{n} \rightarrow 0 \quad$ as $n \rightarrow \infty$. Since, by Proposition 1 (ii), $A_{0} \supset A_{1} \supset \cdots \supset A_{f}$, where $A_{f}$ is the attractor of the IFS, we have $\lambda\left(A_{f}\right)=0$. Since $A_{f}$ is also the support of the 
invariant distribution, $\mu_{f}$, we clearly have $\mu_{f}\left(A_{f}\right)=1$. Thus, $\mu_{f}$ is singular with respect to Lebesgue measure.

The distribution function corresponding to $\mu_{f}$, when $\alpha=1 / 3$, is usually called a Cantor function (since its support is precisely the Cantor ternary set) or a Lebesgue function (since it was used by Lebesgue to demonstrate the need for absolute continuity in the fundamental theorem of calculus). The graph of the distribution function, corresponding to $\mu_{f}$, for $\alpha \in$ $(0,1 / 2)$ is referred to as the "Devil's Staircase" (see Figure 2).

\subsection{Singular Invariant Distributions of the Hellinger Type}

Consider now the case where the technological parameter $\alpha=1 / 2$. In this case, the invariant distribution function, $F$, is the unique ${ }^{7}$ distribution function over $[0,1]$ satisfying the functional equation:

$$
F(x)=\left\{\begin{array}{lr}
p F(2 x) & \text { for } x \in[0,1 / 2) \\
p+(1-p) F(2 x-1) & \text { for } x \in[1 / 2,1]
\end{array}\right.
$$

If $p=1 / 2$, then it is easy to see that $F(x)=x$ for all $x \in[0,1]$ satisfies (13), and so the invariant distribution function, $F$, must be the uniform distribution. In the optimal growth literature, this particular case is discussed in [45].

For $p \neq 1 / 2$, the function, $F$, that is generated by (13) has been extensively discussed in the mathematics literature as an example of a continuous, strictly increasing function, for which the derivative (which exists Lebesgue almost everywhere) vanishes almost everywhere. This necessarily means that $F$ represents a measure that is singular with respect to the Lebesgue measure.

As Brown [11] points out, this type of function is properly attributed to Hellinger [30] in whose thesis it first appears. The function, $F$, is obtained as the limit of a sequence of piecewise linear functions, and the relevant properties of $F$ are then verified from the properties of the converging sequence of functions. Hellinger's construction, which was subsequently generalized by Salem [54], is discussed in detail in Riesz and Sz-Nagy [52], Hewitt and Stromberg [31] and Asplund and Bungart [2]. It is also discussed, from the probabilistic viewpoint, in Billingsley [8].

\footnotetext{
${ }^{7}$ Uniqueness can be shown through the standard IFS argument. As a matter of fact, the graph of $F$ is the invariant set of two linear contraction maps from $[0,1]^{2}$ to itself, defined by $(x, y) \longmapsto[(1 / 2) x, p y]$ and $(x, y) \longmapsto$ $[(1 / 2) x+1 / 2,(1-p) y+p]$.
} 
De Rham [13] deduced the properties of $F$ directly from the functional equation (13) governing $F$, but his presentation is quite terse, as is quite clear from the more comprehensive account of it in Yamaguti, Hata and Kigami [61].

Takacs [58] developed a method consisting in writing down a function, $F$, in explicit form, as an infinite series, and verifying that it is continuous, strictly increasing and singular with respect to Lebesgue measure. Given this result, one can show that the solution of (13) is singular by simply verifying that his $F$ satisfies (13).

Given any $x \in[0,1]$, we can write it uniquely as

$$
x=\sum_{r=0}^{\infty} 2^{-a_{r}}
$$

where $a_{0}<a_{1}<\cdots<a_{r}<\cdots$ are positive integers ${ }^{8}$. Define $\delta=(1 / p)-1$, so that $\delta>0$, and $\delta \neq 1$ (since $p \neq 1 / 2$ ). Now define for $x$ given by (14),

$$
F(x)=\sum_{r=0}^{\infty} \delta^{r}(1+\delta)^{-a_{r}}
$$

and $F(0)=0$. Then, by the theorem of Takacs [58], $F$ is strictly increasing, continuous, and its derivative is zero Lebesgue almost everywhere in the interval $[0,1]$. It remains to verify that $F$ satisfies (13).

For $x=0$, we have $F(x)=p F(2 x)$, as required in (13). Next, pick any $x \in(0,1 / 2]$. Then, $a_{0} \geq 2$ in (14), and

$$
2 x=\sum_{r=0}^{\infty} 2^{-\left(a_{r}-1\right)}
$$

with $a_{0}-1<a_{1}-1<\cdots<a_{r}-1<\cdots$ positive integers. Thus, by definition (15), we have $F(2 x)=\sum_{r=0}^{\infty} \delta^{r}(1+\delta)^{-\left(a_{r}-1\right)}=(1+\delta) F(x)=(1 / p) F(x)$ which verifies (13). Finally, pick any $x \in(1 / 2,1]$. Then $a_{0}=1, a_{1} \geq 2$ in $(14)$, and so by (16),

$$
(2 x-1)=\sum_{r=0}^{\infty} 2^{-\left(a_{r+1}-1\right)}
$$

with $a_{1}-1<a_{2}-1<\cdots<a_{r}-1<\cdots$ positive integers. Thus, by definition (15), we have

$$
p+(1-p) F(2 x-1)=\frac{1}{1+\delta}+\sum_{r=0}^{\infty} \delta^{r+1}(1+\delta)^{-a_{r+1}}=\sum_{r=0}^{\infty} \delta^{r}(1+\delta)^{-a_{r}}=F(x)
$$

which verifies (13).

A graph of $F$ is indicated in Figure 3.

${ }^{8}$ Note that (14) corresponds to the dyadic expansion of $x$, i.e., the representation in base- 2 of $x$, where all multiple representations (such that they are all zero after a certain point) are eliminated. 


\subsection{Mutually Singular Distributions}

In Section 3.2 we have seen that the invariant probability measure of our IFS can be constructed as the image probability of the Bernoulli space $(\Omega, \mathcal{F}, P)$ through the map $\Pi_{\alpha}: \Omega \rightarrow[0,1]$ defined in $(11)$ as $\Pi_{\alpha}(\omega)=(1-\alpha) \sum_{t=0}^{\infty} \omega_{t} \alpha^{t}$. We now use this fact to prove that for all $\alpha \in(0,1 / 2]$, two invariant measures are mutually singular whenever different probabilities $p$ and $p^{\prime}$ are adopted in the IFS. (For the standard definition of mutual singularity, see [6], p. 374 ). The idea is to "transfer" this property from the space $(\Omega, \mathcal{F}, P)$ (on which it is well-known to be true) to the image measure of $\Pi_{\alpha}$.

For each $\omega \in \Omega$ and each $n \in \mathbb{N}$, define

$$
N(\omega, n)=\#\left\{0 \leq t \leq n-1: \omega_{t}=1\right\}
$$

Let $q=(1-p)$ and consider the set $S(q) \subset \Omega$ of sequences such that the frequency of 1 's is $q$ :

$$
S(q)=\left\{\omega \in \Omega: \lim _{n \rightarrow \infty} \frac{N(\omega, n)}{n}=q\right\} .
$$

Of course, $S(q) \cap S\left(q^{\prime}\right)=\varnothing$ for $q \neq q^{\prime}$. By the strong law of large numbers (see [8], p.70), it follows that for each $p \in(0,1), P(S(1-p))=1$. As a consequence, each pair of Bernoulli probabilities $P$ and $P^{\prime}$ over $(\Omega, \mathcal{F})$ generated by marginal probabilities $(\operatorname{Pr}(0)=p, \operatorname{Pr}(1)=1-p)$ and $\left(\operatorname{Pr}(0)=p^{\prime}, \operatorname{Pr}(1)=1-p^{\prime}\right)$ respectively, with $p \neq p^{\prime}$, are mutually singular.

To stress dependence of the invariant measure on parameter $p$, let us denote the image measure of $\Pi_{\alpha}$ (our invariant distribution) by $\mu_{f}^{p}$.

For each $\alpha \in(0,1)$, we can introduce a metric, $\rho_{\alpha}$, on the space $\Omega$, given by

$$
\rho_{\alpha}\left(\omega, \omega^{\prime}\right)=\alpha^{\left|\omega \wedge \omega^{\prime}\right|}
$$

where $\left|\omega \wedge \omega^{\prime}\right|=\min \left\{t: \omega_{t} \neq \omega_{t}^{\prime}\right\}$.

Since, for all $\alpha \in(0,1)$ and all pairs $\omega, \omega^{\prime} \in \Omega$,

$$
\left|\Pi_{\alpha}(\omega)-\Pi_{\alpha}\left(\omega^{\prime}\right)\right| \leq(1-\alpha) \alpha^{\left|\omega \wedge \omega^{\prime}\right|} \sum_{t=0}^{\infty} \alpha^{t}=\rho_{\alpha}\left(\omega, \omega^{\prime}\right),
$$

we note that $\Pi_{\alpha}$ is a Lipschitz continuous map from the metric space $\left(\Omega, \rho_{\alpha}\right)$ to the metric $\operatorname{space}([0,1],|\cdot|)$, with Lipschitz constant 1 . Moreover, if we restrict our attention only to $\alpha \in$ $(0,1 / 2)$, a stronger property holds:

$$
\left|\Pi_{\alpha}(\omega)-\Pi_{\alpha}\left(\omega^{\prime}\right)\right| \geq(1-\alpha) \alpha^{\left|\omega \wedge \omega^{\prime}\right|}\left(1-\sum_{t=1}^{\infty} \alpha^{t}\right)=(1-2 \alpha) \rho_{\alpha}\left(\omega, \omega^{\prime}\right),
$$


that is, $\Pi_{\alpha}$ is bi-Lipschitz for $\alpha<1 / 2$. This implies that $\Pi_{\alpha}$ is one-to-one, and thus invertible, for $\alpha \in(0,1 / 2)$.

A special situation occurs when $\alpha=1 / 2$ : in this case $\Pi_{\alpha}$ is "almost" one-to-one. To see this, note that when $\alpha=1 / 2, \Pi_{\alpha}$ is the base- 2 representation of some real number $x \in[0,1]$ :

$$
x=\sum_{r=0}^{\infty} 2^{-a_{r}} \approx 0 . \omega_{0} \omega_{1} \omega_{2} \ldots
$$

where $a_{0}<a_{1}<\cdots<a_{r}<\cdots$ are positive integers [corresponding to values of $\omega_{t}$ in (11) equal to one]. By dropping double representations, we get a (restricted) one-to-one map. For our purposes, it is useful to note that the inverse is not continuous but certainly measurable.

The discussion above is enough to prove the main result of this section.

Proposition 2 For each $\alpha \in(0,1 / 2]$ and all $p \in(0,1)$, the invariant distributions $\mu_{f}^{p}$ are all mutually singular.

Proof. We have seen that for $\alpha \in(0,1 / 2]$, the map $\Pi_{\alpha}$ is invertible. If $\alpha<1 / 2$, it is a consequence of bi-Lipschitz property. If $\alpha=1 / 2$, we can suitably restrict the domain by dropping a countable set of $P$-measure zero. The inverse map $\Pi_{\alpha}^{-1}$ is measurable. Take two Bernoulli probabilities over $\Omega, P$ and $P^{\prime}$ generated by $p$ and $p^{\prime}$, with $p \neq p^{\prime}$. By the discussion at the beginning of this section, $P$ and $P^{\prime}$ are mutually singular; that is, two sets $\Omega_{1}, \Omega_{2}$ exist so that $\Omega=\Omega_{1} \cup \Omega_{2}, \Omega_{1} \cap \Omega_{2}=\varnothing$ and $P\left(\Omega_{1}\right)=P^{\prime}\left(\Omega_{2}\right)=0$. Consider the images of these two sets through $\Pi_{\alpha}$ : clearly, $\Pi_{\alpha}\left(\Omega_{1}\right)$ and $\Pi_{\alpha}\left(\Omega_{2}\right)$ are measurable, disjoint and cover $\Pi_{\alpha}(\Omega)$. Since $\mu_{f}^{p}\left(\Pi_{\alpha}\left(\Omega_{1}\right)\right)=P\left(\Omega_{1}\right)=0$ and $\mu_{f}^{p^{\prime}}\left(\Pi_{\alpha}\left(\Omega_{2}\right)\right)=0$, the two image measures are mutually singular.

Note that, through a different argument, for $\alpha=1 / 2$ we have established singularity of the Hellinger distribution functions discussed in Section 4.2. As a matter of fact, since for $p=1 / 2$ the (uniform) invariant distribution is itself the Lebesgue measure on [0,1], by Proposition 2, for all $p \neq 1 / 2$ the invariant distribution must be singular with respect to Lebesgue measure.

\section{A Class of Singular Distributions for Higher Values of $\alpha$}

We now carry on our analysis by investigating the behavior of the invariant distribution for values $\alpha>1 / 2$. From Figure 1(b), it is clear that, due to the overlapping of images of the IFS, 
the study of the invariant distribution becomes more difficult.

This section is confined to a partial extension of the results on singularity developed before. It turns out that for the combination of parameter values $(\alpha, p)$ satisfying

$$
1 / 2<\alpha<p^{p}(1-p)^{(1-p)}
$$

the invariant distribution is necessarily singular. Coupled with singularity results already obtained in the previous sections for $0<\alpha<1 / 2$ (plus case $\alpha=1 / 2$ and $p \neq 1 / 2$ ), we can thus assert that for all pairs $(\alpha, p)$ such that

$$
0<\alpha<p^{p}(1-p)^{(1-p)}
$$

the invariant distribution is singular with respect to Lebesgue measure.

This result leads to a general observation on existence of singular invariant distributions in our context. When the entropy of the Bernoulli process, $-(p \ln p+(1-p) \ln (1-p)$ ) (see [7]), is relatively "low", which in our context occurs when it is less than $\ln (1 / \alpha)$, then the invariant distribution is singular. Since the highest value of the entropy is $\ln 2$, this condition is automatically satisfied when $\alpha<1 / 2$. In the special case $\alpha=1 / 2$, the entropy is smaller than $\ln (1 / \alpha)$ for all $p$ except $p=1 / 2$, and so the condition is satisfied for all $p \neq 1 / 2$. Thus, the results of Sections 4.1 and 4.2 can be viewed as particular cases of this general observation.

It is important to remark that singularity below the entropy curve is a well known property in the mathematical literature, linking entropy and dimension theory. For our case of two possibilities, with probabilities $p$ and $(1-p)$, it follows from the analysis of Besicovitch[5]. Eggleston [18] generalized the result of Besicovitch to the case of any finite number of possibilities. A general version of the statement above can be established, using the Shannon-McMillan-Breiman Theorem. This approach is followed in Billingsley [6].

Here we provide a simplified proof without resorting directly to properties of the entropy. We make use of only the notion of Hausdorff dimension, which we briefly review in Section 5.1. In Section 5.2, we return to the sequence space introduced in Section 3.2, and show how the Hausdorff dimension of a "probability 1 set" can be computed to be less than one. This, in turn, means that the Lebesgue measure of this set is zero, and leads to the singularity of the invariant distribution. 


\subsection{Hausdorff Dimension}

Let $(X, d)$ be a metric space. For $F \subset X$, and $s \in \mathbb{R}_{+}$, the s-dimensional Hausdorff outer measure is denoted by $\mathcal{H}^{s}(F ; d)$ and is defined as follows. A collection $\mathcal{A}$ of subsets of $X$ is called a countable cover of $F$ if

$$
F \subset \bigcup_{A \in \mathcal{A}} A
$$

and $\mathcal{A}$ is a countable family of sets. Given any $\delta>0$, a countable cover $\mathcal{A}$ of $F$ is a $\delta$-cover if $|A|_{d} \leq \delta$ for all $A \in \mathcal{A}$, where $|A|_{d}$ denotes the diameter of $A$ :

$$
|A|_{d}=\sup _{x, y \in A} d(x, y)
$$

We define

$$
\mathcal{H}_{\delta}^{s}(F ; d)=\inf \sum_{A \in \mathcal{A}}^{s}\left(|A|_{d}\right)^{s}
$$

where the infimum is taken over all countable $\delta$-covers $\mathcal{A}$ of the set $F . \mathcal{H}_{\delta}^{s}(F ; d)$ is non-increasing in $\delta$, and one can define:

$$
\mathcal{H}^{s}(F ; d)=\lim _{\delta \rightarrow 0} \mathcal{H}_{\delta}^{s}(F ; d)
$$

as the $s$-dimensional Hausdorff outer measure of $F$ (given the metric $d$ ).

A useful result on Hausdorff outer measures is that for $\mathbb{R}$ (with the usual distance), the one-dimensional Hausdorff outer measure of any set coincides with the Lebesgue outer measure of that set (see, for example, [8], p. 208, or [16], p. 148-149).

Suppose $\delta<1$, then, given any $\delta$-cover $\mathcal{A}$, for any pair $s<t$,

$$
\sum_{A \in \mathcal{A}}\left(|A|_{d}\right)^{t}=\sum_{A \in \mathcal{A}}\left(|A|_{d}\right)^{s}\left(|A|_{d}\right)^{t-s} \leq \delta^{t-s} \sum_{A \in \mathcal{A}}\left(|A|_{d}\right)^{s}
$$

and so $\mathcal{H}^{t}(F ; d) \leq \delta^{t-s} \mathcal{H}^{s}(F ; d)$. Hence, $\mathcal{H}^{t}(F ; d)>0$ implies $\mathcal{H}^{s}(F ; d)=\infty$ and $\mathcal{H}^{s}(F ; d)<\infty$ implies $\mathcal{H}^{t}(F ; d)=0$. This means that $\mathcal{H}^{s}(F ; d)$ is decreasing in $s$ and is everywhere $+\infty$ or 0 but, at most, at a single point $s_{0} \geq 0$, where it may satisfy $0<\mathcal{H}^{s_{0}}(F ; d)<\infty$. This argument leads to a useful definition of dimension. The Hausdorff-Besicovitch dimension of $F$ is given by

$$
\operatorname{dim}(F ; d)=\inf \left\{s: \mathcal{H}^{s}(F ; d)=0\right\}=\sup \left\{s: \mathcal{H}^{s}(F ; d)=\infty\right\}
$$

Clearly, if $s_{0} \equiv \operatorname{dim}(F ; d)<\infty$, then for all $s>s_{0}, \mathcal{H}^{s}(F ; d)=0$; and if $s_{0} \equiv \operatorname{dim}(F ; d)>0$, then for all $0 \leq s<s_{0}, \mathcal{H}^{s}(F ; d)=\infty$. 
The relationship between $s$-dimensional Hausdorff outer measure and Hausdorff dimension of a set can be depicted as in Figure 4 .

A useful result (see [23] or [16]) on Hausdorff dimension is the following. Let $(X, d)$ and $\left(Y, d^{\prime}\right)$ be metric spaces, and $f$ a Lipschitz continuous map from $X$ to $Y$. Then, for every $F \subset X$,

$$
\operatorname{dim}\left(f(F) ; d^{\prime}\right) \leq \operatorname{dim}(F ; d)
$$

\subsection{Singularity for Low Entropy}

Recall the probability space $(\Omega, \mathcal{F}, P)$ discussed in Section 3.2, the metric $\rho_{\alpha}$ defined in $(18)$ and the map $\Pi_{\alpha}$ defined in (11). We will be concerned, in particular, with $\rho_{\alpha}$ for $\alpha \in(1 / 2,1)$, and with $\rho_{1 / 2}$, which (for convenience) we will denote by $\rho$.

We have (deliberately) included the metric being used in our definition (and notation) of the Hausdorff dimension, since this dimension clearly varies with the metric. From this perspective, our choice of metric $\rho_{\alpha}$ for the space $\Omega$ is not accidental. Since $\rho_{\alpha}$ depends on $\alpha$ [that is, on the "degree of overlapping" of the images of the maps $f_{0}$ and $f_{1}$ in (9)], it turns out that the dimension of subsets of $\Omega$ depends on the contraction ratio of our IFS. This will play a crucial role in the main result of this section. The following relation indicates how the dimension varies as we employ different metrics $\rho_{\alpha}$, that is, different contraction coefficients $\alpha$.

Let $F \subset \Omega$, and $0<\alpha<1$ be given. Then we have the relation

$$
\operatorname{dim}\left(F ; \rho_{\alpha}\right)=\left[\frac{\ln (1 / 2)}{\ln \alpha}\right] \operatorname{dim}(F ; \rho) .
$$

To see this, denote $[\ln (1 / 2) / \ln \alpha]$ by $b$, and note that for $s \in \mathbb{R}_{+}$, and $\mathcal{A}$ a $\delta$-cover of $F$,

$$
\mathcal{H}_{\delta}^{s b}\left(F ; \rho_{\alpha}\right)=\inf \sum_{A \in \mathcal{A}}\left(|A|_{\rho_{\alpha}}\right)^{s b}=\inf \sum_{A \in \mathcal{A}}\left(|A|_{\rho}\right)^{s}=\mathcal{H}_{\delta}^{s}(F ; \rho)
$$

Thus, letting $\delta \rightarrow 0$, we get

$$
\mathcal{H}^{s b}\left(F ; \rho_{\alpha}\right)=\mathcal{H}^{s}(F ; \rho)
$$

and (20) follows consequently.

Recall from Section 4.3 that $\Pi_{\alpha}$ is Lipschitz with Lipschitz constant 1 . Thus, we have for every $F \subset \Omega$,

$$
\operatorname{dim}\left(\Pi_{\alpha}(F) ;|\cdot|\right) \leq \operatorname{dim}\left(F ; \rho_{\alpha}\right) .
$$


We will now use again the set $S(q) \subset \Omega$ defined in (17). We will show that (by the law of large numbers) $S(q)$ is a set of $P$-measure 1 and of Hausdorff dimension less than 1 whenever condition (19) holds.

If we consider the set $S(q)$ in the metric space $(\Omega, \rho)$ [recall that $\rho_{1 / 2}$ is denoted by $\rho$ ], then we have the Besicovitch-Eggleston-Good result ${ }^{9}$ that

$$
\operatorname{dim}(S(q) ; \rho)=\frac{(p \ln p+q \ln q)}{\ln (1 / 2)}
$$

Using (20) and (22) we have

$$
\operatorname{dim}\left(S(q) ; \rho_{\alpha}\right)=\frac{(p \ln p+q \ln q)}{\ln (\alpha)}
$$

It follows that, under (19),

$$
\operatorname{dim}\left(S(q) ; \rho_{\alpha}\right)<1
$$

Using (21) and (23), we obtain

$$
\operatorname{dim}\left(\Pi_{\alpha}(S(q)),|\cdot|\right)<1 .
$$

By definition of Hausdorff dimension, (24) implies that

$$
\mathcal{H}^{1}\left(\Pi_{\alpha}(S(q)),|\cdot|\right)=0
$$

and since $\mathcal{H}^{1}$ coincides with Lebesgue outer measure, $\lambda^{*}$, we have

$$
\lambda^{*}\left(\Pi_{\alpha}(S(q))\right)=0
$$

In particular then $\Pi_{\alpha}(S(q))$ is a Lebesgue measurable set (in the sense of Caratheodory); see [53], p. 58. There is then a Borel set, $B \subset[0,1]$, such that $\Pi_{\alpha}(S(q)) \subset B$, and $\lambda^{*}(B)=0$; see Proposition 5, p. 58 in [53]. Thus $\lambda(B)=0$, where $\lambda$ is Lebesgue measure on $\mathbb{R}$.

Now, we will show that the Borel set $B$ obtained above has $\mu_{f}(B)=1$, where $\mu_{f}$ is the invariant distribution of our IFS. To see this, note that, by the strong law of large numbers, $P(S(q))=1$. By continuity of $\Pi_{\alpha}$ on $\left(\Omega, \rho_{\alpha}\right), \Pi_{\alpha}^{-1}(B)$ is a Borel set of $\Omega$, and $\Pi_{\alpha}^{-1}(B) \supset S(q)$. Thus,

$$
\mu_{f}(B)=P\left(\Pi_{\alpha}^{-1}(B)\right) \geq P(S(q))=1
$$

\footnotetext{
${ }^{9}$ This important result was established for the case of two possibilities by Besicovitch[5]. For an arbitrary finite number of possibilities, it was conjectured by Good [28], and proved by Eggleston [18]. For alternative approaches, see Billingsley ([6], [7]), and Edgar [16]. Note that $(p \ln p+q \ln q)$ measures the negative of the metric entropy of a Bernoulli process with probabilities $p$ and $q$. Therefore, the ratio on the right side of (22) is the metric entropy relative to its maximum value $\ln 2$.
} 
Since $B$ is a Borel set in $[0,1]$ with $\lambda(B)=0$ and $\mu_{f}(B)=1, \mu_{f}$ is singular with respect to Lebesgue measure. We have thus demonstrated the following.

Proposition 3 For $1 / 2<\alpha<p^{p}(1-p)^{1-p}$, $\mu_{f}$ is singular with respect to Lebesgue measure.

\section{Absolutely Continuous Invariant Distributions}

It remains to examine properties of the invariant distribution for pairs $(\alpha, p)$ satisfying $(1)$ :

$$
p^{p}(1-p)^{1-p}<\alpha<1
$$

As we have already indicated in Section 3.3, for $p=1 / 2$ and for Lebesgue almost every $\alpha \in(1 / 2,1)$, Solomyak [55] showed that the distribution has density in $L^{2}$. His original proof was based on Fourier transforms and the problem of estimating multiple zeros of certain power series. The method of Fourier transform applies naturally to the symmetric case $p=1 / 2$ and proves useful in developing a theory in $L^{2}$.

Subsequently, Peres and Solomyak [50] developed an alternative method, based on differentiation of measures instead of Fourier transforms, to prove the same theorem as in [55]. The latter approach (by-passing $L^{2}$ theory) actually opened a way for some extensions to the asymmetric case $p \neq 1 / 2$, where $L^{2}$ theory fails to capture the main features of the invariant distribution in some circumstances.

The main results along this direction are worked out by Peres and Solomyak [51] and may be summarized as follows. By confining the range of values for the probability $p$ to the interval $[1 / 3,2 / 3]$, the authors established that the invariant distribution $\mu_{f}$ is generically absolutely continuous above the entropy curve, namely for almost every $p^{p}(1-p)^{1-p}<\alpha<1$. Furthermore, again for $1 / 3 \leq p \leq 2 / 3, \mu_{f}$ turns out to exhibit gradually increasing smoothness as the technological parameter $\alpha$ rises above the entropy curve, more specifically, for $1<\gamma \leq 2$ and for almost every $\left[p^{\gamma}+(1-p)^{\gamma}\right]^{1 /(\gamma-1)} \leq \alpha<1, \mu_{f}$ has density in $L^{\gamma}$. Note that a theory only in $L^{2}$ would be far too stringent to investigate this last behavior.

This seems to be the present state of art, at least pertaining to a characterization in terms of (generic) absolute continuity above the entropy curve. More work is needed to extend the above statements to values of $p$ outside the interval $[1 / 3,2 / 3]$; perhaps, as indicated by Peres and Solomyak, a new approach may be needed. 
However, another result in [51], stated in terms of necessary smoothness condition, sheds some more light on this "gradually increasing smoothness" of $\mu_{f}$ for higher values of $\alpha$. The authors, indeed, state that for all $p$ and all $\gamma>1$, if $\mu_{f}$ is absolutely continuous with density in $L^{\gamma}$, then necessarily $\alpha \geq\left[p^{\gamma}+(1-p)^{\gamma}\right]^{1 /(\gamma-1)}$.

In this section, we confine ourselves to a brief description of the basic ingredients necessary to deal with our specific problem. We provide some material on Fourier Transforms in Section 6.1, while Section 6.2 shows how this material works in Solomyak's [55] construction for the symmetric case and how some method of estimating multiple zeros of power series can be used. Finally, Section 6.3 discusses the extensions to the case $p \neq 1 / 2$ obtained in [51].

\subsection{Fourier Transforms of the Erdös Series}

Instead of looking at the invariant distribution $\mu_{f}$, of our IFS, or equivalently the invariant distribution of the Erdös series (11) discussed in Section 3.2, we find it convenient to work with a class of series that includes (12). We describe this (class of) Erdös series as follows.

Let $J \subset \mathbb{Z}_{+}$and $\varepsilon_{j}$ (for $j \in J$ ) are independent and identically distributed random variables taking values on the set $D=\{-1,+1\}$, with $P\left(\varepsilon_{j}=-1\right)=p$ and $P\left(\varepsilon_{j}=+1\right)=q$. Suppose $n$ is a positive integer such that $j \in J$ if and only if $j+n \in J$. Define $\ell=\#(J \cap[0, n-1])$; then $J$ is a union of $\ell$ arithmetic progressions with difference equal to $n$. For example, with $J=\mathbb{Z}_{+}$, we have $n=1$ and $\ell=1$; with $J=\{0,1,3,4,6,7,9, \ldots\}$, every third integer is omitted, so $n=3$ and $\ell=2$.

We define the random variable

$$
Y_{\alpha}^{J}=\sum_{j \in J} \varepsilon_{j} \alpha^{j}
$$

Note that this is the same as the random variable defined in (12), when $J=\mathbb{Z}_{+}$. However, we need also to consider "rarefied" versions of the Erdös series here, which is the reason for defining the class of Erdös series given by (25). For $J=\mathbb{Z}_{+}$, we denote $Y_{\alpha}^{J}$ by $Y_{\alpha}$.

Given $J$ and $\alpha$, let us denote the distribution of $Y_{\alpha}^{J}$ by $\eta$. Its Fourier transform is given (as the function of the real variable, $s$ ) by:

$$
\phi_{\eta}(s)=\int e^{i s x} \eta(d x)=\mathbb{E}\left[e^{i s Y_{\alpha}^{J}}\right]=\mathbb{E}\left[\prod_{j \in J} e^{i s \varepsilon_{j} \alpha^{j}}\right]
$$


By independence of $\varepsilon_{j}$, it is easy to obtain

$$
\phi_{\eta}(s)=\prod_{j \in J} \mathbb{E}\left[e^{i s \varepsilon_{j} \alpha^{j}}\right]=\prod_{j \in J}\left[p e^{-i \alpha^{j} s}+q e^{i \alpha^{j} s}\right] .
$$

Working with the Fourier transform of $\eta$ rather than $\eta$ itself is primarily motivated, in the current context, by the "inversion formula". In particular, if

$$
\int_{-\infty}^{\infty}\left|\phi_{\eta}(s)\right| d s<\infty
$$

then the distribution $\eta$ has a continuous density, given by

$$
f(x)=(1 / 2 \pi) \int_{-\infty}^{\infty} e^{-i s x} \phi_{\eta}(s) d s .
$$

See Billingsley [8], p. 296-304, for the basic properties of Fourier transforms of probability measures (known as characteristic functions) on the real line.

Since $\left|\phi_{\eta}(s)\right| \leq 1$ for all $s \in \mathbb{R}$, if $(27)$ holds $\left(\phi_{\eta} \in L^{1}\right)$, we also have

$$
\int_{-\infty}^{\infty}\left|\phi_{\eta}(s)\right|^{2} d s<\infty
$$

so that $\phi_{\eta} \in L^{2}$. Thus, a natural question is whether (28) itself can ensure the absolute continuity of $\eta$. This is indeed possible, as shown by the " $L^{2}$-theory" of Fourier transforms. [See, for example, Chung [12], p. 159]. Briefly, if $\phi_{\eta}$ satisfies (28), then by Plancherel's theorem (see, for example, Feller [25], p. 599-601), there exists $f \in L^{2}$ such that:

$$
\int_{0}^{x} f(u) d u=\frac{1}{\sqrt{2 \pi}} \int_{0}^{\infty}\left(\frac{e^{i s x}-1}{-i s}\right) \phi_{\eta}(s) d s
$$

Now, one can use the inversion formula to show that

$$
F(x)-F(0)=\frac{1}{\sqrt{2 \pi}} \int_{0}^{x} f(u) d u
$$

where $F$ is the distribution function, corresponding to the distribution $\eta$.

In view of this, if one can show that for every $\varepsilon>0$

$$
\int_{a+\varepsilon}^{b-\varepsilon}\left[\int_{-\infty}^{\infty}\left|\phi_{\eta}(s)\right|^{2} d s\right] d \alpha<\infty
$$

where $a<b$, then it would follow that for Lebesgue almost every $\alpha \in(a, b),(28)$ holds, and so the distribution $\eta$ is absolutely continuous, with $L^{2}$ density.

The results of Solomyak [55], which we discuss in Section 6.2, provides sufficient conditions under which (29) is satisfied, for certain specifications of $a$ and $b$. 


\subsection{The Result of Solomyak in the Symmetric case $p=1 / 2$}

The result of Solomyak [55] that solves the Erdös problem in the symmetric case is the following.

Theorem 1 (Solomyak) For $p=1 / 2$, the distribution of $Y_{\alpha}$ has a density in $L^{2}(\mathbb{R})$ for Lebesgue almost every $\alpha \in(1 / 2,1)$.

The proof of this theorem rests upon a more general result, which establishes a sufficient condition for $\left(L^{2}\right)$ absolute continuity based on the existence of a certain type of power series.

Proposition 4 For $p=1 / 2$, the distribution of $Y_{\alpha}^{J}$ has $L^{2}(\mathbb{R})$ density for a.e. $\alpha \in\left((1 / 2)^{\ell / n}, \tau\right)$ provided

$$
(1 / 2)^{\ell / n}<\tau
$$

where $\tau \in(0,1)$ is the smallest number for which there exists a power series $g(x) \not \equiv 0$,

$$
g(x)=\sum_{j \in J} g_{j} x^{j}, \quad \text { where } g_{j} \in\{-1,0,1\}
$$

with $g(\tau)=g^{\prime}(\tau)=0$.

The specifications required at the end of the last section in order to apply Fourier transforms theory as in (29), are indeed $a=(1 / 2)^{\ell / n}$ and $b=\tau$. To be more precise, existence of such power series is a sufficient condition for the following transversality condition to hold on the interval $(a, b) \subset(0,1)$. Say that the transversality condition holds on $(a, b)$ for a given pair $\omega, \omega^{\prime} \in \Omega$ if

$$
\alpha \longmapsto \Pi_{\alpha}(\omega)-\Pi_{\alpha}\left(\omega^{\prime}\right) \quad \text { has no double zero on }(a, b) \text { for } \omega \neq \omega^{\prime}
$$

Here a "double zero" for the difference $f(\alpha)=\Pi_{\alpha}(\omega)-\Pi_{\alpha}\left(\omega^{\prime}\right)$ means $\alpha_{0}$ such that $f\left(\alpha_{0}\right)=$ $f^{\prime}\left(\alpha_{0}\right)=0$. The graphic interpretation is that the graphs of functions $\alpha \longmapsto \Pi_{\alpha}(\omega)$ and $\alpha \longmapsto \Pi_{\alpha}\left(\omega^{\prime}\right)$ restricted to the interval $(a, b)$, intersect transversally (if at all).

In order to apply Proposition 4 to obtain Theorem 1, one has to have an efficient method of isolating "multiple zeros" of power series. Solomyak himself calculates some lower bounds for such zeros that we shall now exploit to see how Proposition 4 and the use of Fourier transforms yield Theorem 1 .

The first step in proving Theorem 1 is to find an interval $(a, b) \subset(0,1)$ such that the transversality condition holds. We shall see that such an interval is $(a, b)=\left((1 / 2)^{\ell / n}, \tau\right)=$ 
$\left(1 / 2,2^{-1 / 2}\right)$; then Proposition 4 states that for a.e. $\alpha \in\left(1 / 2,2^{-1 / 2}\right)$, the distribution $\mu_{f}$ is absolutely continuous with $L^{2}$ density. In order to fill the whole interval $(1 / 2,1)$, one must rely on convolutions through Fourier transform as in (26). Let us see this procedure in some detail.

Take $J=\mathbb{Z}_{+}$, so that $\ell=n=1$ and $(\ell / n)=1$ in Proposition 4 . Thus, the first step tackles directly our Erdös series. Solomyak finds a lower estimation $\tau>0.649$ for this case. Hence, in view of Proposition 4, the distribution $\mu_{f}$ of $Y_{\alpha}$ has density in $L^{2}(\mathbb{R})$ for almost every $\alpha \in(1 / 2,0.649)$.

Next, consider the "rarefied" Erdös series so that every third integer from $Z_{+}$is omitted, namely, let $J=\{0,1,3,4,6,7,9, \ldots\}$, so that $n=3$ and $\ell=2$. By Solomyak's calculations, the number $\tau$ for this rarefied series satisfies $\tau>0.713$. Since $2^{-2 / 3} \approx 0.630<0.713$, Proposition 4 (for $\ell / n=2 / 3$ ) is nonempty and thus for a.e. $\alpha \in\left(2^{-2 / 3}, 0.713\right)$ the distribution $\eta$ of $Y_{\alpha}^{J}$ has $L^{2}$ density. In this case, its Fourier transform $\phi_{\eta} \in L^{2}$ and since $\left|\phi_{\mu_{f}}\right| \leq\left|\phi_{\eta}\right|$, we also have $\phi_{\mu_{f}} \in L^{2}$. That is, we have found that for a.e. $\alpha \in(1 / 2,0.649) \cup\left(2^{-2 / 3}, 0.713\right), \mu_{f}$ has $L^{2}$ density. Note, however, that these two open intervals overlap, as $2^{-2 / 3} \approx 0.630<0.649$. Moreover, $2^{-1 / 2} \approx 0.707<0.713$. Therefore, we conclude that for a.e. $\alpha \in\left(1 / 2,2^{-1 / 2}\right)$, $\mu_{f}$ has $L^{2}$ density.

The second step consists in extending this finding to a.e. $\alpha \in\left(2^{-1 / k}, 2^{-1 / 2 k}\right)$ for $k=2,3, \ldots$. Then, since $2^{-1 / k} \rightarrow 1$ as $k \rightarrow \infty$, it follows that $\mu_{f}$ has $L^{2}$ density for a.e. $\alpha \in(1 / 2,1)$. We demonstrate this for $k=2$, the rest following by induction. For a.e. $\alpha \in\left(2^{-1 / 2}, 2^{-1 / 4}\right)$, we have the Fourier transform of $\eta$ given by (26), which yields

$$
\phi_{\eta}^{\alpha}(s)=\phi_{\eta}^{\alpha^{2}}(s) \phi_{\eta}^{\alpha^{2}}(\alpha s)
$$

Since $\alpha \in\left(2^{-1 / 2}, 2^{-1 / 4}\right)$ implies $\alpha^{2} \in\left(1 / 2,2^{-1 / 2}\right)$, we have each of the two Fourier transforms on the right hand side of (30) in $L^{2}$, and so by Hölder's inequality $\phi_{\eta}^{\alpha}$ is in $L^{1}$. Since $\phi_{\eta}^{\alpha}$ is also in $L^{\infty}$, we have $\phi_{\eta}^{\alpha} \in L^{1}(R) \cap L^{\infty}(\mathbb{R}) \subset L^{2}(\mathbb{R})$.

Finally, by induction, one can check that for a.e. $\alpha \in \bigcup_{k=0}^{\infty}\left(2^{-1 / k}, 2^{-1 / 2 k}\right), \phi_{\eta}^{\alpha} \in L^{2}(\mathbb{R})$, and so $\eta$ has $L^{2}$ density. Thus, $\mu_{f}$ is absolutely continuous with $L^{2}$ density for a.e. $\alpha \in(1 / 2,1)$.

\subsection{An Extension of Solomyak Theorem to the Asymmetric Case} $p \neq 1 / 2$

The main findings in [51] for the asymmetric case pertaining to our IFS are the following. 


\section{Theorem 2 (Peres - Solomyak)}

i) If $1 / 3 \leq p \leq 2 / 3$, then for almost every $p^{p}(1-p)^{1-p}<\alpha<1 \mu_{f}$ is absolutely continuous;

ii) If $1 / 3 \leq p \leq 2 / 3$ and $1<\gamma \leq 2$, then for almost every $\left[p^{\gamma}+(1-p)^{\gamma}\right]^{1 /(\gamma-1)} \leq \alpha<1 \mu_{f}$ has density in $L^{\gamma}$;

iii) for all $0<p<1$ and all $\gamma>1$, if $\mu_{f}$ is absolutely continuous with density in $L^{\gamma}$, then $\alpha \geq\left[p^{\gamma}+(1-p)^{\gamma}\right]^{1 /(\gamma-1)}$.

The meaningful innovation in the proof of both parts $(i)$ and $(i i)$ with respect to the technique adopted in the proof of Theorem 1, is the use of the Radon-Nykodym derivative of the invariant measure, $\partial \mu_{f} / \partial x$, which actually is its density and exists whenever $\mu_{f}$ is absolutely continuous. The importance of this method lies in by-passing $L^{2}$ theory ${ }^{10}$ which relies on (29). As a matter of fact, in place of (29) the authors show that

$$
\int_{a}^{b}\left[\int_{-\infty}^{\infty}\left(\underline{D}\left(\mu_{f}, x\right)\right)^{\gamma-1} d \mu_{f}(x)\right] d \alpha<\infty,
$$

where

$$
\underline{D}\left(\mu_{f}, x\right)=\liminf _{r \rightarrow 0^{+}}(2 r)^{-1} \mu_{f}\left[B_{r}(x)\right]
$$

and $B_{r}(x)$ denotes the ball of center $x$ and radius $r$, holds for any interval $(a, b) \subset(0,1)$ such that $a>\left[p^{\gamma}+(1-p)^{\gamma}\right]^{1 /(\gamma-1)}$ and the transversality condition holds. As for the upper extremum of interval $(a, b)$, they use the same estimation as in [55]: $b=0.649<\tau$, which clearly works since, for $1 / 3 \leq p \leq 2 / 3$ and $\gamma \leq 2$,

$$
\left[p^{\gamma}+(1-p)^{\gamma}\right]^{1 /(\gamma-1)} \leq p^{2}+(1-p)^{2} \leq(1 / 3)^{2}+(2 / 3)^{2}=5 / 9<0.649
$$

Hence, part $(i)$ of Theorem 2 is true for $p^{p}(1-p)^{1-p}<\alpha<0.649$, and the extension to the whole interval $\left(p^{p}(1-p)^{1-p}, 1\right)$ [and to interval $\left[\left[p^{\gamma}+(1-p)^{\gamma}\right]^{1 /(\gamma-1)}, 1\right)$ in part $\left.(i i)\right]$ is established through convolution techniques similar to that discussed in Section 6.2.

Actually slightly more can be said: since the transversality condition holds for all pairs $(\alpha, p)$ such that $\alpha \leq 0.649$, part $(i)$ of Theorem 2 it is immediately extended for values of $p$ in the larger interval $(0.156,0.844)$ [where the extrema are the solutions of the equation

\footnotetext{
${ }^{10}$ Note that this approach includes the $L^{2}$ case for $\gamma \geq 2$, since $\left[p^{\gamma}+(1-p)^{\gamma}\right]^{1 /(\gamma-1)}=p^{2}+(1-p)^{2}$ for $\gamma=2$. However, a theory purely in $L^{2}$, like the one pursued in [55], would have missed absolute continuity of $\mu_{f}$ (with density in $L^{\gamma}$ for $\left.1<\gamma<2\right)$ between $p^{p}(1-p)^{1-p}$ and $p^{2}+(1-p)^{2}$.
} 
$\left.p^{p}(1-p)^{1-p}=0.649\right]$ and for $p^{p}(1-p)^{1-p}<\alpha<0.649$. A similar argument also applies to part $(i i)$ of Theorem 2 , therefore yielding the following corollary.

\section{Corollary 1}

i) If $0.156<p<0.844$, then for almost every $p^{p}(1-p)^{1-p}<\alpha<0.649 \mu_{f}$ is absolutely continuous;

ii) If $0.156<p<0.844$ and $1<\gamma \leq 2$ is such that $\left[p^{\gamma}+(1-p)^{\gamma}\right]^{1 /(\gamma-1)}<0.649$, then for almost every $\left[p^{\gamma}+(1-p)^{\gamma}\right]^{1 /(\gamma-1)} \leq \alpha<0.649 \mu_{f}$ has density in $L^{\gamma}$.

The difficulty in extending the last result for values of $p \in(0.156,0.844)$ outside the interval $[1 / 3,2 / 3]$ and for values of $\alpha$ larger than 0.649 lies in the use of convolutions techniques, which do not work in this case.

Necessary smoothness condition ( $i i i)$ in Theorem 2 is derived from a theorem of HardyLittlewood [29].

\section{A Bifurcation Diagram}

It is clear that in the equal probability case, we are able to describe the nature of the stochastic steady-state for (Lebesgue) almost every value of the technological parameter, $\alpha$. However, in the asymmetric case $(p \neq 1 / 2)$, the results of Sections 4,5 and 6 are unable to completely characterize the invariant distribution, especially for values of technological parameter $\alpha$ above the entropy curve and values of probability $p$ outside the interval $[1 / 3,2 / 3]$.

In view of all known results reviewed in the previous sections, we are able to sketch only a partial "bifurcation diagram" that includes the combinations of parameter values covered by our analysis. It appears as in figure 5.

In view of necessary smoothness condition expressed in part (iii) of Theorem 2 , it is natural to formulate a conjecture for the missing values of $\alpha$ above the entropy curve and $p$ outside interval $[1 / 3,2 / 3]$. In $(i i i)$ is excluded the possibility that $\mu_{f}$ is too smooth below curve $\left[p^{\gamma}+(1-p)^{\gamma}\right]^{1 /(\gamma-1)}$, namely, given that $\mu_{f}$ has density in $L^{\gamma}$, then $\alpha \geq\left[p^{\gamma}+(1-p)^{\gamma}\right]^{1 /(\gamma-1)}$, for any $\gamma>1$. Note that $\lim _{\gamma \rightarrow 1^{+}}\left[p^{\gamma}+(1-p)^{\gamma}\right]^{1 /(\gamma-1)}=p^{p}(1-p)^{(1-p)}$ and the graph of function $\left[p^{\gamma}+(1-p)^{\gamma}\right]^{1 /(\gamma-1)}$ moves upward away from the graph of the entropy curve $p^{p}(1-p)^{1-p}$ for 
increasing values of $\gamma$, until converging to the graph of function $\max (p, 1-p)$, that is, $\lim _{\gamma \rightarrow+\infty}$ $\left[p^{\gamma}+(1-p)^{\gamma}\right]^{1 /(\gamma-1)}=\max (p, 1-p)$.

The conjecture, thus, is that for all $0<p<1$, as one moves upward above the entropy curve, $\mu_{f}$ is generically absolutely continuous with density becoming gradually better shaped, starting from having density in $L^{1}$ (close to the entropy curve), then having density in $L^{2}$ for $\gamma \geq 2$ [this is already known to be true by part $(i i)$ of Theorem 2 at least for $p \in[1 / 3,2 / 3$ ]] and eventually having an essentially bounded density for $\gamma \rightarrow+\infty$, that is, for almost every $\max (p, 1-p) \leq \alpha<1$. This situation is depicted in figure 6 .

However, since very few cases of singular measures above the entropy curve are known (actually the only known examples are those given by Erdös for $p=1 / 2$, see [20]), even if the portrait in figure 6 were true, there is still room for investigating Garsia's [27] much stronger conjecture that the invariant measure is absolutely continuous for all $p^{p}(1-p)^{(1-p)}<\alpha<1$ but a countable set of points.

\section{Appendix}

In this final section we present a result connecting $L$-metric convergence to weak convergence of measures, which we used in our exposition in Section 2.1.2.

Proposition 5 Convergence in the L-metric defined in (6) implies weak convergence over $\mathcal{L}(X)$; that is, given any sequence $\mu_{t}$ in $\mathcal{L}(X)$ and a measure $\mu_{f}$ in $\mathcal{L}(X), L\left(\mu_{t}, \mu_{f}\right) \rightarrow 0$ implies $\mu_{t} \Longrightarrow \mu_{f}$.

To prove this result we need the following lemma which is a variant of Uryshon Lemma.

Lemma 1 Let $(X, d)$ be a metric space and $A, B \subset X$ non-empty closed disjoint sets. If $d(A, B)>0$, then there exists a Lipschitz function $g: X \rightarrow \mathbb{R}$ such that $g(a)=0$ for all $a \in A$, $g(b)=1$ for all $b \in B$ and $0<g(x)<1$ for all $x \notin A \cup B$.

Proof. Consider

$$
g(x)=\frac{d(x, A)}{d(x, A)+d(x, B)}
$$

Since $d(A, B)>0$, there is $\delta$ such that $d(x, A)+d(x, B)>\delta>0$. Hence, $g$ satisfies all requirements but Lipschitz property. To see that $g$ is Lipschitz, for any $x, y \in X$ and $\varepsilon>0$, 
find $a \in A$ so that $d(a, y) \leq d(y, A)+\varepsilon$. Then

$$
d(x, A) \leq d(x, a) \leq d(x, y)+d(y, a) \leq d(x, y)+d(y, A)+\varepsilon
$$

and

$$
d(x, A)-d(y, A) \leq d(x, y)+\varepsilon
$$

Since $\varepsilon$ is arbitrary, and by exchanging $x$ with $y$, we get

$$
|d(x, A)-d(y, A)| \leq d(x, y)
$$

from which follows

$$
|g(x)-g(y)| \leq \delta^{-1} d(x, y)
$$

Proof of Proposition 5. It suffices to show that

$$
\limsup _{t \rightarrow \infty} \mu_{t}(C) \leq \mu_{f}(C)
$$

for all closed sets $C \subset X$.

Note that

$$
L\left(\mu_{t}, \mu_{f}\right) \rightarrow 0 \Longrightarrow \int g d \mu_{t} \rightarrow \int g d \mu_{f}
$$

for any Lipschitz function $g$. Take any closed (proper) subset $C \subset X$ and consider the sequence of open sets $\left\{C_{1 / n}\right\}$, where $C_{\delta}$ denotes the $\delta$-parallel body of $C$ defined in (8). Clearly $C \subset C_{1 / n}$ for all $n$ and the complement $C_{1 / n}^{c}$ is non-empty for $n$ large enough. By Lemma 1 there exists a Lipschitz function $g_{n}$ such that $g_{n}(x)=1$ for $x \in C$ and $g_{n}(x)=0$ for $x \in C_{1 / n}^{c}$. Therefore

$$
\mu_{t}(C) \leq \int g_{n} d \mu_{t} \leq \mu_{t}\left(C_{1 / n}\right)
$$

and

$$
\limsup _{t \rightarrow \infty} \mu_{t}(C) \leq \int g_{n} d \mu_{f} \leq \mu_{f}\left(C_{1 / n}\right)
$$

which, for $n \rightarrow \infty$, gives (31). 


\section{References}

[1] Alexander, J. and J. Yorke, Fat Baker's Transformation, Journal of Ergodic Theory and Dynamical Systems 4: 1-23, 1984.

[2] Asplund, E. and L. Bungart, A First Course in Integration, New York: Holt, Rinehart and Winston, 1966.

[3] Barnsley, M. F., Fractals Everywhere, Boston: Academic Press, 1988.

[4] Barnsley, M. F. and S. Demko, Iterated Function Systems and the Global Construction of Fractals, Proceeding Royal Society London Series A 399: 243-275, 1985.

[5] Besicovitch, A. S., On the Fundamental Geometric Properties of Linearly Measurable Plane Sets of Points II, Math. Annalen 115: 296-329, 1938.

[6] Billingsley, P., Hausdorff Dimension in Probability Theory, Illinois Journal of Mathematics 4: 187-209, 1960.

[7] Billingsley, P., Ergodic Theory and Information, New York: John Wiley, 1965.

[8] Billingsley, P., Probability and Measure, New York: John Wiley \& Sons, 1979.

[9] Brock, W. A. and M. Majumdar, Global Asymptotic Stability Results for Multisector Models of Optimal Growth under Uncertainty When Future Utilities are Discounted, Journal of Economic Theory 18: 225-243, 1978.

[10] Brock, W. A. and L. J. Mirman, Optimal Economic Growth and Uncertainty: the Discounted Case, Journal of Economic Theory 4: 479-513, 1972.

[11] Brown, A., An Elementary Example of a Continuous Singular Function, American Mathematical Monthly 81: 295-297, 1969.

[12] Chung, K. L., A Course in Probability Theory, New York: Academic Press, 1974.

[13] De Rham, G., Sur Quelques Courbes Définies par des Équations Functionelles, Rendiconti dei Seminari di Matematica dell'Università di Torino 16: 101-113, 1957; English translation reprinted as "On Some Curves Defined by Functional Equations" in G.A. Edgar, ed., Classics on Fractals, New York: Addison-Wesley, 285-297, 1993. 
[14] Donaldson, J. B. and R. Mehra, Stochastic Growth with Correlated Production Shocks, Journal of Economic Theory 29: 282-312, 1983.

[15] Dubins, L. E. and D. A. Freedman, Invariant Probabilities for Certain Markov Processes, Annals of Mathematical Statistics 37: 837-838, 1966.

[16] Edgar, G. A., Measure, Topology and Fractal Geometry, New York: Springer-Verlag, 1990.

[17] Edgar, G. A., An Erdös Problem, Supplemento ai Rendiconti del Circolo Matematico di Palermo, Serie II 28: 439-440, 1992.

[18] Eggleston, H. G., The Fractional Dimension of a Set Defined by Decimal Properties, Quarterly Journal of Mathematics Oxford Series 20: 31-36, 1949.

[19] Elton, J. H., An Ergodic Theorem for Iterated Maps, Journal of Ergodic Theory and Dynamical Systems 7: 481-488, 1987.

[20] Erdös, P., On a Family of Symmetric Bernoulli Convolutions, American Journal of Mathematics 61: 974-975, 1939.

[21] Erdös, P., On the Smoothness Properties of Bernoulli Convolutions, American Journal of Mathematics 62: 180-186, 1940.

[22] Falconer, K. J., The Hausdorff Dimension of Self-Affine Fractals, Proceedings of the Cambridge Philosophical Society 103: 339-350, 1988.

[23] Falconer, K. J., Fractal Geometry, Chichester: John Wiley \& Sons, 1990.

[24] Falconer, K. J., Techniques in Fractal Geometry, New York: John Wiley \& Sons, 1997.

[25] Feller, W., An Introduction to Probability Theory and its Applications, Volume II, New York: John Wiley \& Sons, 1966.

[26] Futia, C. A., Invariant Distributions and the Limiting Behavior of Markovian Economic Models, Econometrica 50: 377-408, 1982.

[27] Garsia, A. M., Arithmetic Properties of Bernoulli Convolutions, Transactions of the American Mathematical Society 102: 409-432, 1962. 
[28] Good, I. J., The Fractional Dimensional Theory of Continued Fractions, Proceedings of the Cambridge Philosophical Society 37: 199-228, 1941.

[29] Hardy, G. and J. Littlewood, Some Properties of Fractional Integrals, Math. Z. 27: 565606, 1928.

[30] Hellinger, E., Die Orthogonalinvarianten Quadratischer Formen von Unendlichvielen Variabelen (Dissertation), Göttingen, 1907.

[31] Hewitt, E. and K. Stromberg, Real and Abstract Analysis: A Modern Treatment of the Theory of Functions of a Real Variable, Berlin, New York: Springer-Verlag, 1965.

[32] Hopenhayn, H. A. and E. C. Prescott, Stochastic Monotonicity and Stationary Distributions for Dynamic Economies, Econometrica 60: 1387-1406, 1992.

[33] Hutchinson, J., Fractals and Self-similarity, Indiana University Mathematics Journal 30: 713-747, 1981.

[34] Jessen, B. and A. Wintner, Distribution Functions and the Riemann Zeta Function, Transactions of American Mathematical Society 38: 48-88, 1935.

[35] Kershner, R. and A. Wintner, On Symmetric Bernoulli Convolutions, American Journal of Mathematics 57: 541-548, 1935.

[36] Karlin, S., Some Random Walks Arising in Learning Models, Pacific Journal of Mathematics 3: 725-756, 1953.

[37] Lasota, A. and M. C. Mackey, Chaos, Fractals, and Noise, Stochastic Aspects of Dynamics New York: Springer-Verlag, 1994.

[38] Lebesgue, H., Lecons sur l'Intégration et la Recherche des Functions Primitives, Paris: Gauthier-Villars, 1904.

[39] Lucas, R. E. and E. C. Prescott, Investment under Uncertainty, Econometrica 5: 659-681, 1971.

[40] Majumdar, M. and R. N. Bhattacharya, On a Class of Stable Random Dynamical Systems: Theory and Applications, CAE Working Paper 98-08, Cornell University, 1998. 
[41] Mandelbrot, B. B., The Fractal Geometry of Nature, San Francisco: W. H. Freeman, 1982.

[42] Mauldin D. and K. Simon, The Equivalence of some Bernoulli Convolutions to Lebesgue Measure, Proceedings of the American Mathematical Society 126: 2733-2736, 1998.

[43] Mirman, L. J., On the Existence of Steady States Measures for One Sector Growth Models with Uncertain Technology, International Economic Review 13: 271-286, 1972.

[44] Mirman, L. J., The Steady State Behavior of a Class of One Sector Growth Models with Uncertain Technology, Journal of Economic Theory 6: 219-242, 1973.

[45] Mirman, L. J. and I. Zilcha, On Optimal Growth under Uncertainty, Journal of Economic Theory 11: 329-339, 1975.

[46] Montrucchio, L. and F. Privileggi, Fractal Steady States in Stochastic Optimal Control Models, Annals of Operations Research 88: 183-197, 1999.

[47] Norman, M. F., Some Convergence Theorems for Stochastic Learning Models with Distance Diminishing Operators, Journal of Mathematical Psychology 5: 61-101, 1968.

[48] Peres, Y., and W. Schlag, Smoothness of Projections, Bernoulli Convolutions and the Dimension of Exceptions, Duke Mathematical Journal 102: 193-251, 2000.

[49] Peres, Y., W. Schlag and B. Solomyak, Sixty Years of Bernoulli Convolutions, in Bandt, Graf and Zahle, eds., Fractal Geometry and Stochastics II, Birkhauser Verlag, 2000.

[50] Peres, Y. and B. Solomyak, Absolute Continuity of Bernoulli Convolutions, a Simple Proof, Mathematical Research Letters 3: 231-239, 1996.

[51] Peres, Y. and B. Solomyak, Self-Similar Measures and Intersections of Cantor Sets, Transactions of the American Mathematical Society 350: 4065-4087, 1998.

[52] Riesz, F. and B. Sz-Nagy, Functional Analysis, New York: Ungar, 1955.

[53] Royden, H. L., Real Analysis, New York: MacMillan, 1988.

[54] Salem, R., On some Singular Monotonic Functions which are Strictly Increasing, Transactions of American Mathematical Society 53: 427-439, 1943. 
[55] Solomyak B., On the Random Series $\sum \pm \lambda^{n}$ (an Erdös Problem), Annals of Mathematics 142: 611-625, 1995.

[56] Stark, J. and P. Bressloff, Iterated Function Systems and their Applications, in M. Farge, J. C. R. Hunt and J. C. Vassilicos, eds., Wavelets, Fractals and Fourier Transforms, Oxford: Clarendon Press, 65-90, 1993.

[57] Stokey, N. L. and R. E. Lucas, Recursive Methods in Economic Dynamics, Cambridge, Mass.: Harvard University Press, 1989.

[58] Takacs, L., An Increasing Continuous Singular Function, American Mathematical Monthly 90: 35-37, 1978.

[59] Vrscay, E. R., Iterated Function Systems: Theory, Applications and the Inverse Problem, in J. Bélair and S. Dubuc, eds., Fractal Geometry and Analysis, Dordrecht: Kluwer Academic Publishers, 405-468, 1991.

[60] Wintner, A., On Convergent Poisson Convolutions, American Journal of Mathematics 57: 827-838, 1935.

[61] Yamaguti, M., M. Hata and J. Kigami, Mathematics of Fractals, Translations of Mathematical Monographs, Vol. 167, American Mathematical Society, Providence, Rhode Island, 1993. 


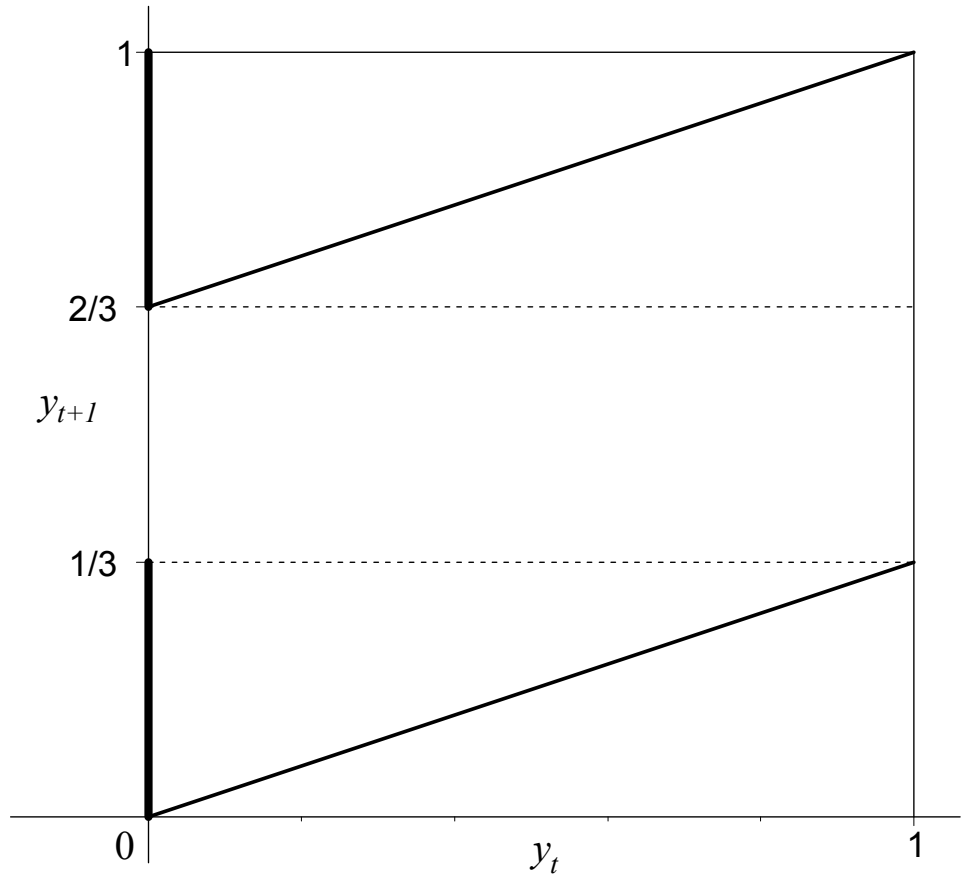

a)

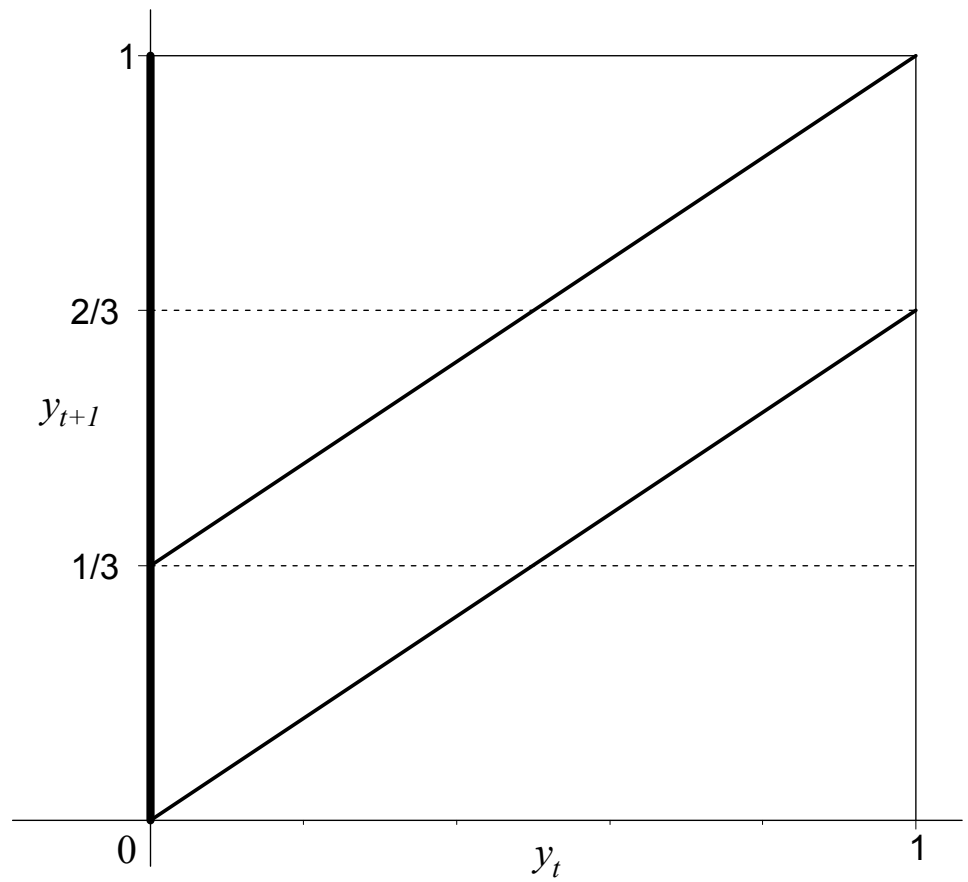

b)

Figure 1: Plot of $y_{t+1}=f_{z}\left(y_{t}\right), z \in\{0,1\}$ : a) $\alpha=1 / 3$, non overlapping images; b) $\alpha=2 / 3$, overlapping images. 


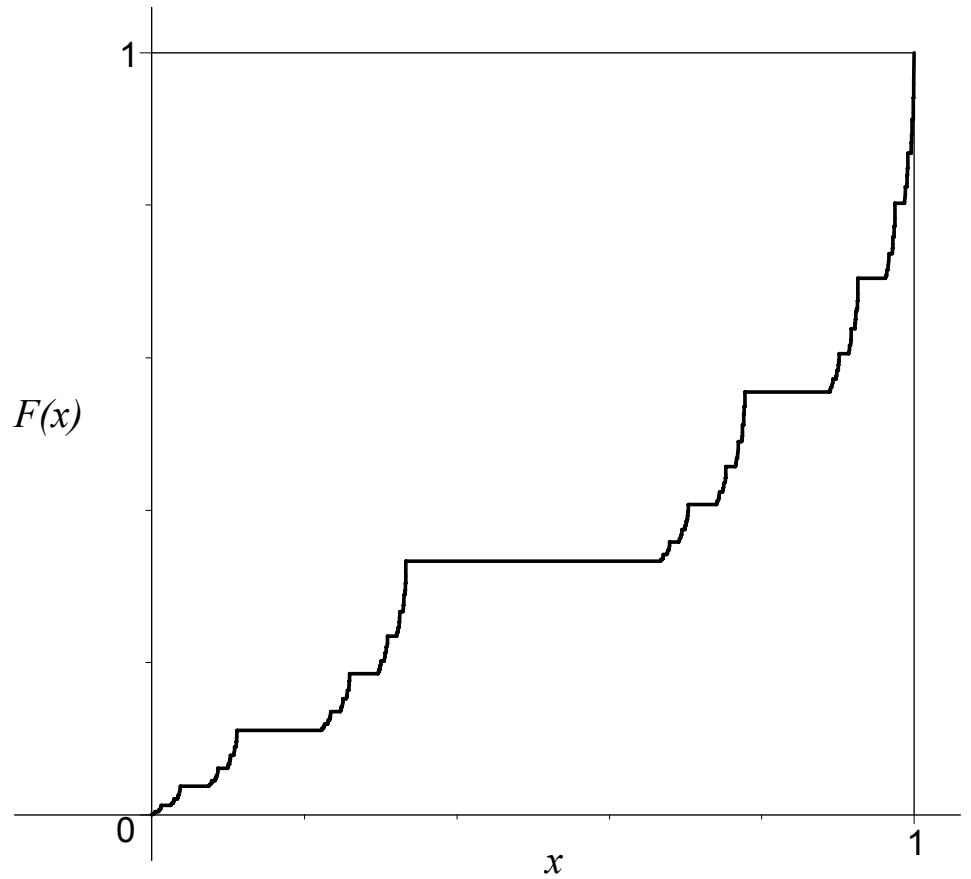

a)

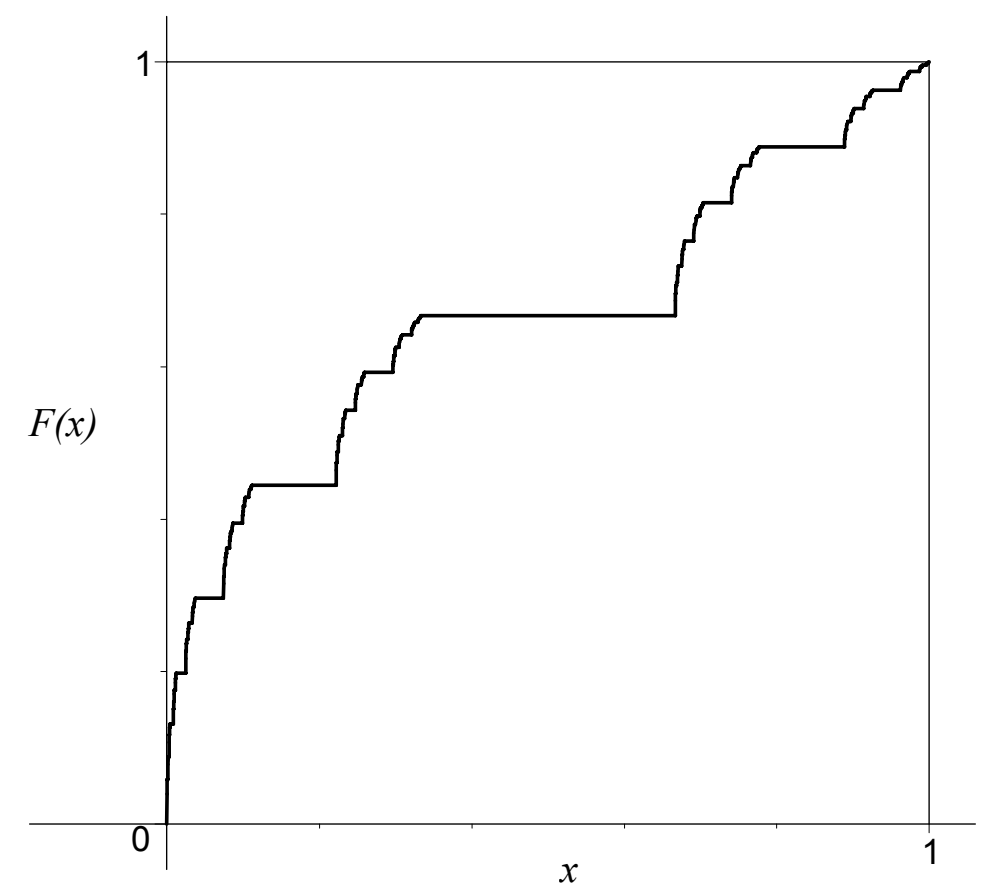

b)

Figure 2: Devil's Staircase: a) $\alpha=1 / 3$ and $p=1 / 3$; b) $\alpha=1 / 3$ and $p=2 / 3$. 


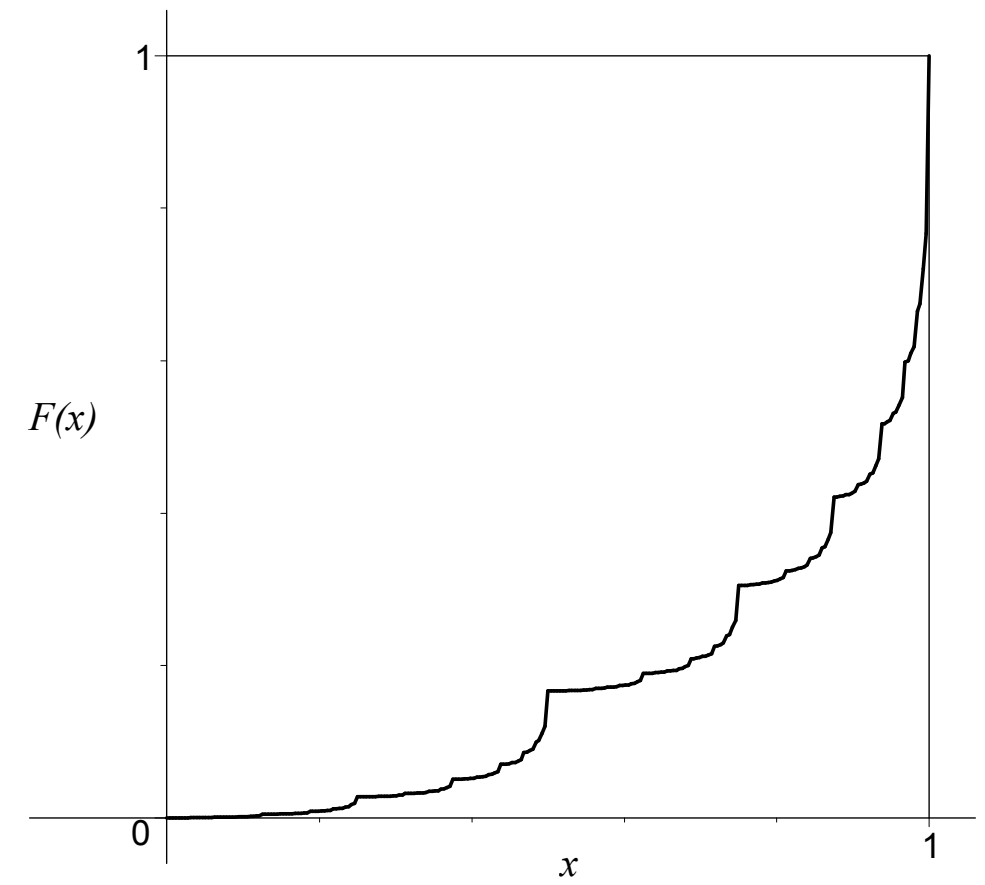

a)

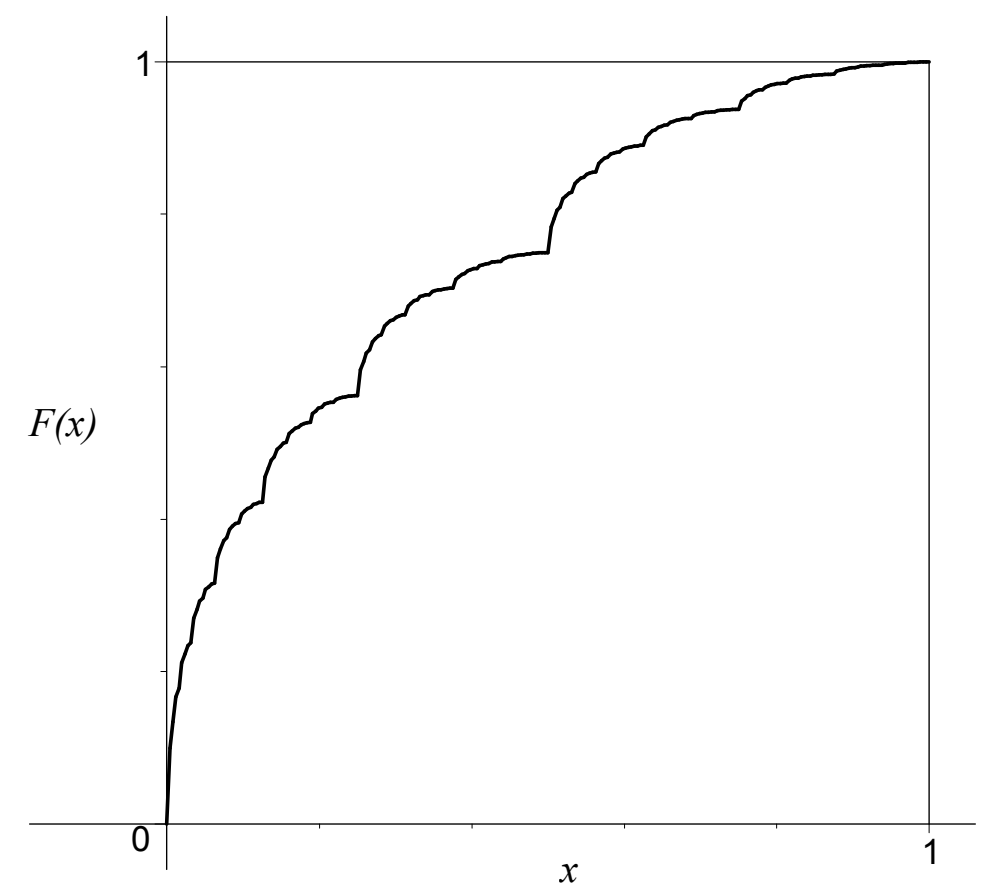

b)

Figure 3: Hellinger distributions: a) $\alpha=1 / 2$ and $p=1 / 6$; b) $\alpha=1 / 2$ and $p=3 / 4$. 


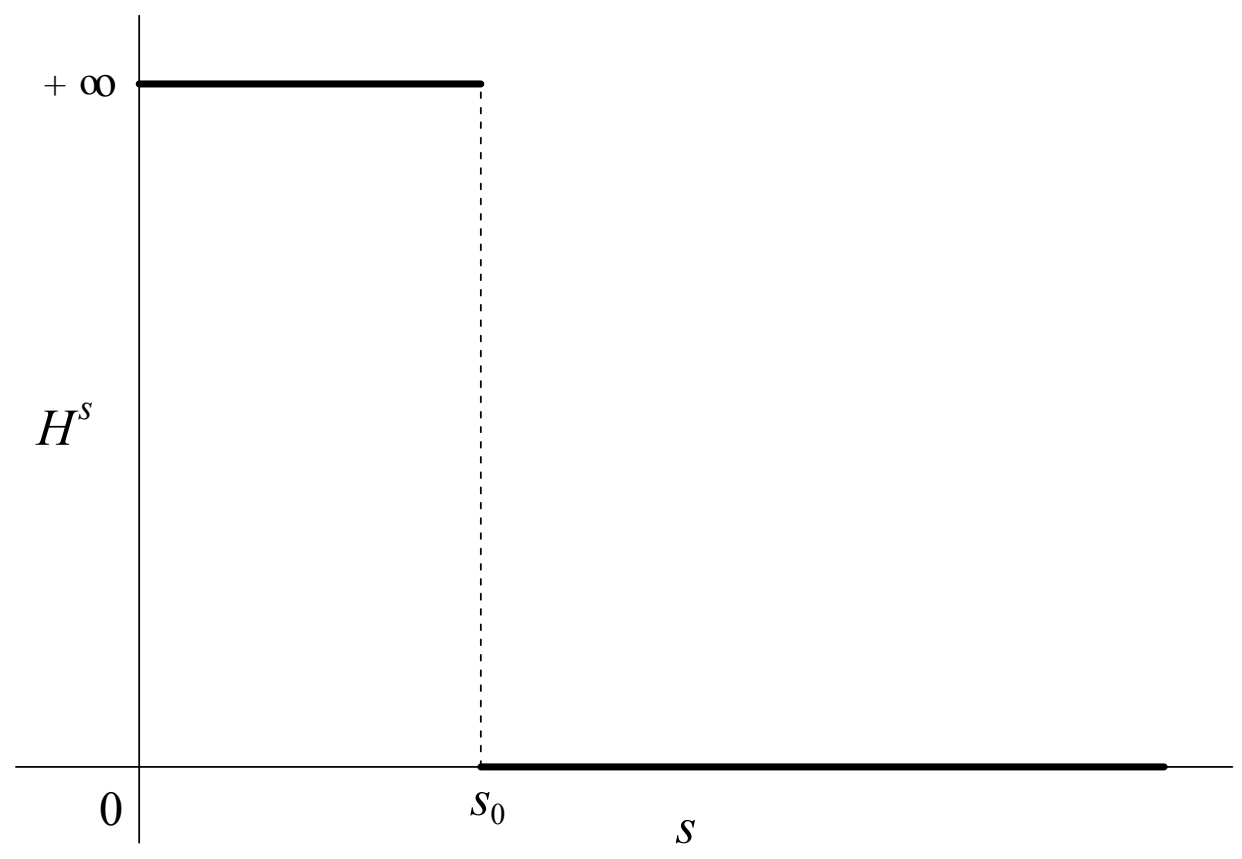

Figure 4: Plot of $\mathcal{H}^{s}(F ; d)$ where $s_{0} \equiv \operatorname{dim}(F ; d)$. 


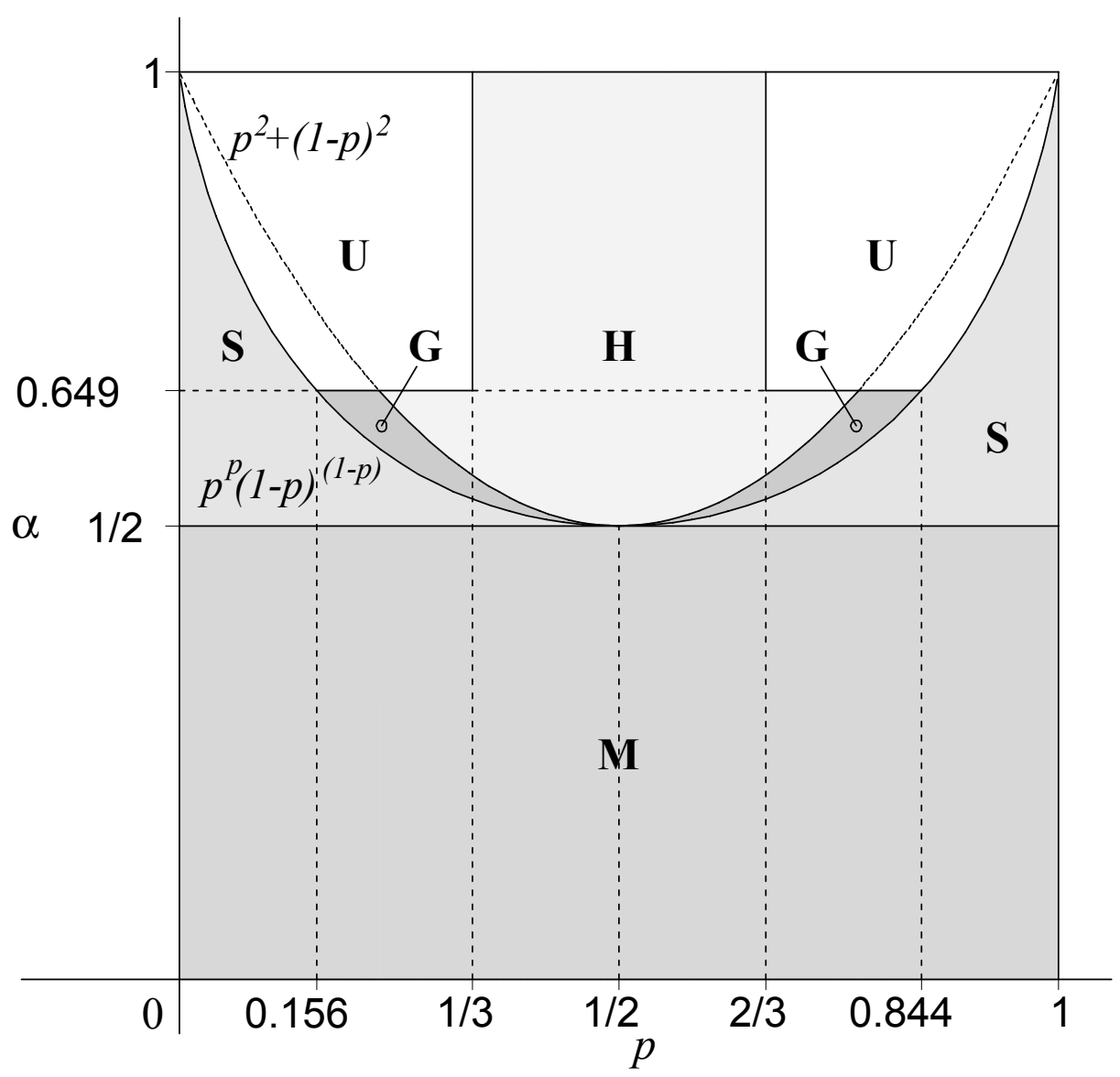

Figure 5: M: mutually singular measures over Cantor sets; $\mathbf{S}$ : measures singular with respect to Lebesgue measure; H: a.e. absolutely continuous measures with density in $L^{2}$; G: a.e. absolutely continuous measures with density in $L^{\gamma}$, with $1<\gamma \leq 2$ [recall that $\left[p^{\gamma}+(1-p)^{\gamma}\right]^{1 /(\gamma-1)}=$ $p^{2}+(1-p)^{2}$ when $\left.\gamma=2\right] ; \mathbf{U}$ : unknown area. 


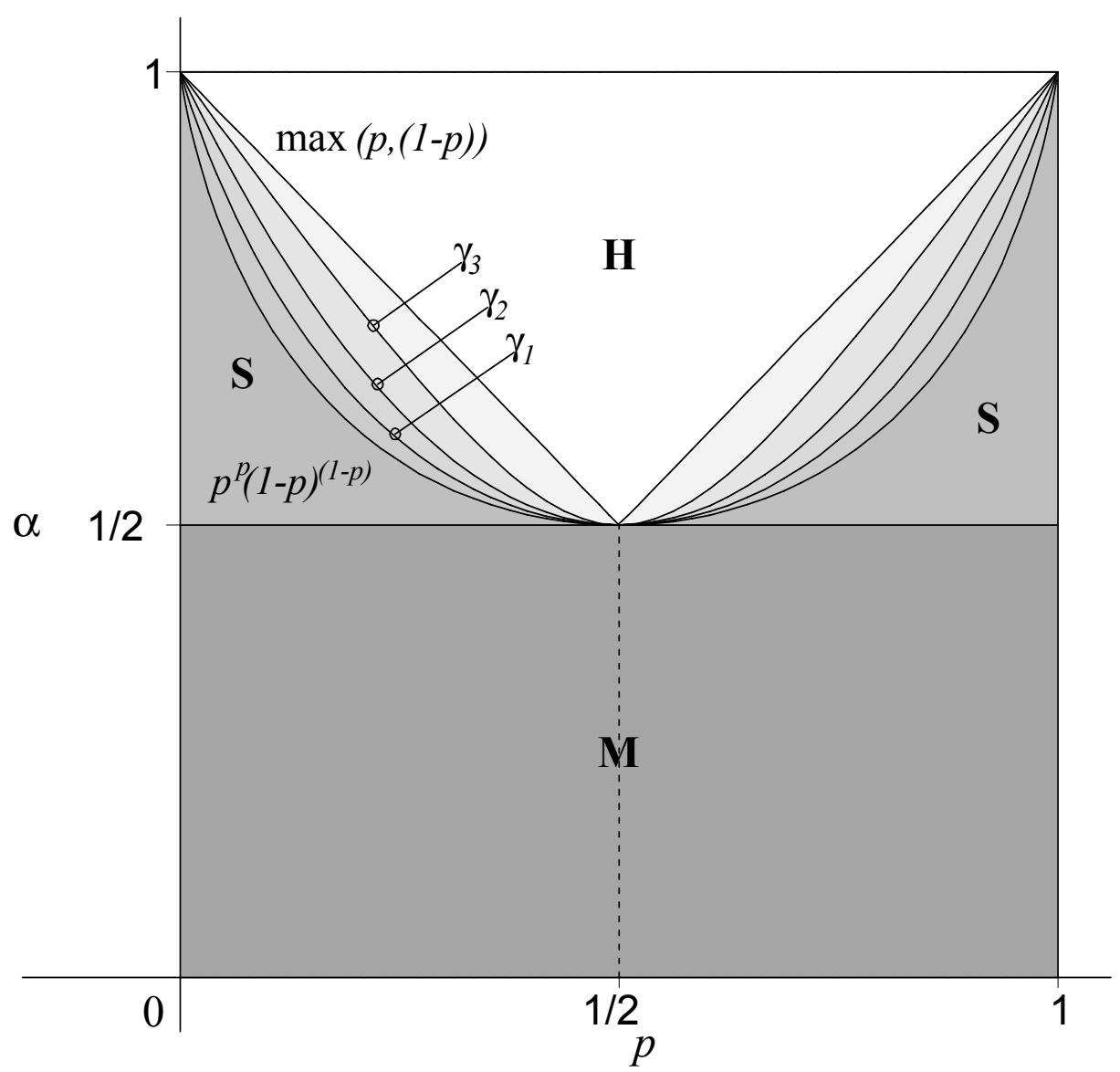

Figure 6: M: mutually singular measures over Cantor sets; $\mathbf{S}$ : measures singular with respect to Lebesgue measure; $\mathbf{H}$ : a.e. absolutely continuous measures with bounded density. In the area between $\mathbf{S}$ and $\mathbf{H}$, the invariant measure has density in $L^{\gamma}, \gamma>1$, depending on $\left[p^{\gamma}+(1-p)^{\gamma}\right]^{1 /(\gamma-1)}$. Three increasingly smooth cases are plotted between the entropy curve and $\max (p, 1-p): \gamma_{1}=11 / 8, \gamma_{2}=2$ and $\gamma_{3}=4$. 